q-alg/9609020

To appear in Rev. Math. Phys.

\title{
On the Structure of Monodromy Algebras and Drinfeld Doubles
}

\author{
FLORIAN NILL* \\ Institut für Theoretische Physik, \\ FU-Berlin, Arnimallee 14, D-14195 Berlin
}

Aug. 23, 1996

Revised Oct. 6, 1996

\begin{abstract}
We give a review and some new relations on the structure of the monodromy algebra (also called loop algebra) associated with a quasitriangular Hopf algebra $H$. It is shown that as an algebra it coincides with the so-called braided group constructed by S. Majid on the dual of $H$. Gauge transformations act on monodromy algebras via the coadjoint action. Applying a result of Majid, the resulting crossed product is isomorphic to the Drinfeld double $\mathcal{D}(H)$. Hence, under the so-called factorizability condition given by $\mathrm{N}$. Reshetikhin and M. Semenov-Tian-Shansky, both algebras are isomorphic to the algbraic tensor product $H \otimes H$. It is indicated that in this way the results of Alekseev et al. on lattice current algebras are consistent with the theory of more general Hopf spin chains given by K. Szlachányi and the author. In the Appendix the multi-loop algebras $\mathcal{L}_{m}$ of Alekseev and Schomerus [AS] are identified with braided tensor products of monodromy algebras in the sense of Majid, which leads to an explanation of the "bosonization formula" of [AS] representing $\mathcal{L}_{m}$ as $H \otimes \ldots \otimes H$.
\end{abstract}

\section{Introduction}

Monodromy algebras and Drinfeld doubles have appeared as quantum symmetry algebras in several models of low dimensional quantum field theory during the last few years.

Monodromy or loop algebras associated with quasitriangular Hopf algebras $H$ play an important rôle in lattice approaches to Chern-Simons theory [AGS], topological quantum field theory [AS] and current algebras on a circle [AFFS]. In these models they commonly appear as algebras generated by the matrix elements of quantum holonomy operators $\mathbf{M}=\left(M_{a b}^{I}\right)$ around closed loops. Their center is spanned by generators obeying the Verlinde algebra and commuting with all other link operators. Their represenation theory has been used in [AGS,AS] to study quantum Chern-Simons algebras ("graph algebras") on Riemann surfaces with coloured

${ }^{*}$ E-mail: NILL@physik.fu-berlin.de Supported by the DFG, SFB 288 "Differentialgeometrie und Quantenphysik" 
punctures. As a particular input result the authors show that under the assumption on $H$ to be modular Hopf algebra the representation category of the associated monodromy algebra coincides with that of $H$ itself [AS].

In [AFFS] these results have been used and further developped to study current algebras on a periodic lattice chain. The authors introduce "gauged loop algebras" $\mathcal{K}$ given as crossed products of a monodromy algebra with a copy of the gauge quantum group sitting on the initial (三 end) point of the loop. It turns out [AFFS] that the center of $\mathcal{K}$ already coincides with the center of the whole lattice current algebra $\mathcal{A}$, thereby governing again its representation theory. Under the same assumptions as above it is proven in [AFFS] that the irreducible representations of $\mathcal{K}$ - and therefore of $\mathcal{A}$ - are in one-to-one correspondence with those of $H \otimes H$. 円

On the other hand, in non-abelian spin systems $[\mathrm{SzV}]$ and their generalizations to Hopf spin sytems [NSz] it is the Drinfeld double $\mathcal{D}(H)$ [Dr] which plays a similarly central rôle. In fact, it appears as the appropriate generalization of the familiar order-disorder symmetry group $G \times G$ known from abelian $G$-spin models. As a rather complete classification result it has been proven in $[\mathrm{NSz}]$ that in the infinite lattice limit the category of DHR-superselection sectors of these models is precisely given by $\operatorname{Rep}(\mathcal{D}(H))$. This has been done by constructing an equivalence class of localized transportable coactions $\rho: \mathcal{A} \rightarrow \mathcal{A} \otimes \mathcal{D}(H)$, where here $\mathcal{A}$ denotes the quasilocal $C^{*}$-algebra generated by all local observables on the infinite lattice chain. One may then show that these coactions are universal in the sense that any charged represenation $\pi_{r}$ of $\mathcal{A}$ satisfying the DHR-selection criterion relative to some Haag-dual vacuum representation $\pi_{0}$ is equivalent to a representation $\left(\pi_{0} \otimes D_{r}\right) \circ \rho$ where $D_{r}$ is a representation of $\mathcal{D}(H)$ and where the correspondence $\pi_{r} \leftrightarrow D_{r}$ is one-to-one on equivalence classes. The associated statistics operators are $\mathbf{C}$-matrix valued and are precisely given by the representation matrices of the canonical quasi-triangular $R$-matrix of $\mathcal{D}(H)$. The above coaction is in a way dual to the symmetry action of $\mathcal{D}(H)$, which leaves the observables invariant and only acts on the orderdisorder fields.

All this confirms with the fact that the Drinfeld double has also been discovered in 2dimensional continuum models to describe the quantum symmetry associated with nonabelian order and disorder (kink) fields [DPR,Mü].

Not obviously being related, Majid has developed his theory of braided groups during the last 5 years [M3-M7] by also constructing new algebra and coalgebra structures from a given quasitriangular Hopf algebra $H$. Particular examples of physical interest are among others the Sklynanin Algebra and the quantum Lorentz group [M5]. As a guiding construction principle, braided groups always appear as (co-)algebras in a category of $H$-modules, i.e. all structural maps are built so as to become $H$-module morphisms. In the cases of interest for us one takes $H$ (or its dual $\hat{H}$ ) itself as the underlying space and considers the (co-)adjoint $\mathrm{H}$-action. The given structures of $H$ and $\hat{H}$ must then be deformed in order to provide $H$-module morphisms under this action. Based on this philosophy one is also naturally lead to depart from ordinary tensor products to braided tensor products [M4]. Majid also provides what he calls "bosonization formulas" [M4,M5,M7,M8] by showing that various crossed product constructions by nontrivial Hopf actions lead to algebras which are nevertheless isomorphic to the ordinary tensor product of its constituents. This might already give a first hint to some hidden relations with the above mentioned results of [AFFS] on the representation theory of their algebra $\mathcal{K}$.

\footnotetext{
${ }^{1}$ This holds true on finite lattices. The discussion about the infinite lattice limit in [AFFS] is heuristic and inconclusive in various respects.
} 
Digging further into the literature one discovers that already in 1988 Reshetikhin and Semenov-Tian-Shansky [RS1] have provided a somewhat related "bosonization formula" by noting that under a what they called "factorizability condition" on the $R$-matrix of a quasitriangular Hopf algebra $H$ the associated Drinfeld double $\mathcal{D}(H)$ is isomorphic to $H \otimes H$ ?. Around the same time Majid had developed his theory of the Drinfeld double as a double crossed product of $H$ with $\widehat{H_{o p}}$. He then discovered that without any additional conditions the Drinfeld double of a quasi-triangular Hopf algebra $H$ is always isomorphic to an ordinary crossed product $\hat{H}_{R} \rtimes H$ [M2] by constructing a Hopf module action of $H$ on a newly invented algebra $\hat{H}_{R}$, which only latter became to be described as the "braided group function algebra" [M4] dual to the braided group introduced in [M3].

Putting all these pieces together one could already arrive at the conjecture that Majid's crossed product $\hat{H}_{R} \rtimes H$ might be the same as the algebra $\mathcal{K}$ in [AFFS], the latter also being a crossed product (i.e. \{monodromy algebra\} $\rtimes H$ ), and that both algebras are therefore isomorphic to the Drinfeld double $\mathcal{D}(H)$. The identification with the representation category of $H \otimes H$ would then be a consequence of the results of [RS1], since their factorizability condition is in fact nothing but the invertibility property of the linear "monodromy map"

$$
\operatorname{mon}_{R}: \hat{H} \ni \varphi \mapsto(\varphi \otimes i d)\left(R_{o p} R\right) \in H
$$

which was proven (although somewhat hiddenly) to hold for modular Hopf algebras in Section 5.2 of $[\mathrm{AS}]$.

This picture may be further supported by the observation, that apart from the periodic boundary condition the lattice current algebra of [AFFS] is in fact a reformulation of the Hopf spin chain of $[\mathrm{NSz}]$. To see this let me shortly review that the latter is defined by placing a copy of a finite dimensional Hopf algebra $\mathcal{A}_{2 i} \cong H$ on each site and a copy of the dual algebra $\mathcal{A}_{2 i+1} \cong \hat{H}$ on each link of a one dimensional lattice. Non-vanishing commutation relations are then postulated only on neighbouring site-link pairs, where one requires [NSz]

$$
\begin{aligned}
& A_{2 i}(a) A_{2 i-1}(\varphi)=A_{2 i-1}\left(\varphi_{(1)}\right)\left\langle\varphi_{(2)} \mid a_{(1)}\right\rangle A_{2 i}\left(a_{(2)}\right) \\
& A_{2 i+1}(\varphi) A_{2 i}(a)=A_{2 i}\left(a_{(1)}\right)\left\langle a_{(2)} \mid \varphi_{(1)}\right\rangle A_{2 i+1}\left(\varphi_{(2)}\right)
\end{aligned}
$$

Here sites (links) are numbered by even (odd) integers and $H \ni a \mapsto A_{2 i}(a) \in \mathcal{A}_{2 i} \subset \mathcal{A}, \hat{H} \ni$ $\varphi \mapsto A_{2 i+1}(a) \in \mathcal{A}_{2 i+1} \subset A$ denote the embeddings of the single site (link) algebras into the global quantum chain $\mathcal{A}$. As usual, $a_{(1)} \otimes a_{(2)} \equiv \sum_{i} a_{(1)}^{i} \otimes a_{(2)}^{i}=\Delta(a)$ denotes the coproduct on $H$ (and analogously on $\hat{H}$ ) and $\langle a \mid \varphi\rangle \equiv\langle\varphi \mid a\rangle$ denotes the dual pairing $H \otimes \hat{H} \rightarrow \mathbf{C}$.

Let now $R \in H \otimes H$ be quasitriangular and put $\mathbf{R}_{2 i}:=\left(i d \otimes A_{2 i}\right)\left(R_{o p}^{-1}\right) \in H \otimes \mathcal{A}_{2 i}$, where $R_{o p}$ denotes the image of $R$ under the permutation of tensor factors. Let us also introduce

$$
\mathbf{L}_{2 i+1}:=\left(i d \otimes A_{2 i+1}\right)(\mathbf{E}) \in H \otimes \mathcal{A}_{2 i+1}
$$

where $\mathbf{E}=\sum e_{\nu} \otimes e^{\nu} \in H \otimes \hat{H}$ is the canonical element given by any basis $e_{\nu} \in H$ with dual basis $e^{\nu} \in \hat{H}$. One may then define "lattice currents"

$$
\mathbf{J}_{2 i+1}:=\mathbf{R}_{2 i}^{-1} \mathbf{L}_{2 i+1} \in H \otimes \mathcal{A}
$$

\footnotetext{
${ }^{2}$ Actually, this is more then just a bosonization, since the copy of the dual algebra $\hat{H} \subset \mathcal{D}(H)$ is traded for a copy of $H$. We will make the relations more explicit by dividing this transformation into two steps in Section 7, see also Chapter 7 of [M8]
} 
which are immediately verified to satisfy the lattice current algebra of [AFFS]

$$
\begin{aligned}
{\left[\mathbf{1}_{H} \otimes A_{2 i}(a)\right] \mathbf{J}_{2 i-1} } & =\mathbf{J}_{2 i-1}\left[a_{(1)} \otimes A_{2 i}\left(a_{(2)}\right)\right] \\
\mathbf{J}_{2 i+1}\left[\mathbf{1}_{H} \otimes A_{2 i}(a)\right] & =\left[a_{(1)} \otimes A_{2 i}\left(a_{(2)}\right)\right] \mathbf{J}_{2 i+1} \\
\mathbf{J}_{2 i+1}^{13} \mathbf{J}_{2 i+1}^{23} & =R^{12}(\Delta \otimes i d)\left(\mathbf{J}_{2 i+1}\right) \\
\mathbf{J}_{2 i-1}^{13} R^{12} \mathbf{J}_{2 i+1}^{23} & =\mathbf{J}_{2 i+1}^{23} \mathbf{J}_{2 i-1}^{13}
\end{aligned}
$$

where the last two lines are understood as identities in $H \otimes H \otimes \mathcal{A}$, the upper indices indicating the canonical embeddings of tensor factors.

Hence, under the additional data given by a quasitriangular $R$ the lattice algebras of [NSz] and $[\mathrm{AFFS}]$ are isomorphic. Moreover, periodic boundary conditions may be imposed in both versions by identifying $\mathcal{A}_{0} \equiv \mathcal{A}_{2 N}$.

The discovery of this isomorphism was the starting motivation for the present work. In fact, it seemed hard to believe that the different results on the representation theory of this model (i.e. isomorphic to $\operatorname{Rep} \mathcal{K} \equiv \operatorname{Rep} H \otimes H$ in [AFFS] and to $\operatorname{Rep} \mathcal{D}(H)$ in [NSz]) should be caused by the different approaches of the authors and/or the different boundary conditions.

As it turns out now, the true explanation is indeed given by the chain of isomorphisms mentioned above

$$
\mathcal{K} \cong \hat{H}_{R} \rtimes H \cong \mathcal{D}(H) \cong H \otimes H
$$

(the last one holding provided $\operatorname{mon}_{R}$ is invertible). Moreover, all of this could in principle be deduced from the literature as quoted, provided one realizes that the braided group function algebra $\hat{H}_{R}$ of Majid is in fact the same object as the monodromy algebra introduced in [AGS,AS,AFFS]. So with this point of view the paper could end with just proving this last statement.

However I would say that even the experts agree that the relevant informations are spread in an rather unrelated way over the literature and often have very different appearances and notations making it hard to compare or even identify them. Due to the importance of these structures in the various mathematical and physical contexts mentioned above I therefore consider it worthwhile to give a selfcontained and unifying account of the whole story here.

So let me start in Section 2 by identifying the monodromy algebras of [AGS,AS,AFFS] as (duals of) braided groups $\hat{H}_{R}$ in the sense of Majid [M4,M5]. In Section 3 I will then review Majid's construction of a coadjoint $H$-action on $\hat{H}_{R}$ and identify the resulting crossed product $\mathcal{M}_{R}(H):=\hat{H}_{R} \rtimes H$ with the gauged monodromy algebra $\mathcal{K}$ given in Definition 5 of [AFFS]. Section 4 gives a complete proof of an observation of [AFFS] on the existence of commuting left and right monodromies inside $\mathcal{M}_{R}(H)$. In the course of this proof we find a copy of $\hat{H}$ naturally embedded as a subalgebra in $\mathcal{M}_{R}(H)$. In this way we recover as a Corollary in Section 5 the isomorphism $\mathcal{M}_{R}(H) \cong \mathcal{D}(H)$ previously obtained in [M2]. In Section 6 we use methods of [M5] to show that the monodromy map mon $_{R}$ extends to a homomorphism of crossed products $\operatorname{Mon}_{R}: \mathcal{M}_{R}(H) \rightarrow H \rtimes_{A d} H$ which becomes an isomorphism iff mon is $_{R}$ bijective (here $A d$ denotes the adjoint action of $H$ on itself). Since by a simple bosonization formula the crossed product $H \rtimes_{A d} H$ is naturally isomorphic to the algebraic tensor product $H \otimes_{\text {alg }} H$, this clarifies the results of [AFFS] on the representation theory of $\mathcal{K} \equiv \mathcal{M}_{R}(H)$ without relying on any semisimplicity assumptions.

In Section 7 we proceed to the Hopf algebra structure induced on $\mathcal{M}_{R}(H) \equiv \mathcal{K}$ by the isomorphism with $\mathcal{D}(H)$ and investigate its image on $H \otimes_{\text {alg }} H \cong H \rtimes_{A d} H$ under the map Mon $_{R}$. We define on $H \otimes_{\text {alg }} H$ a cocycle deformed coproduct $\delta_{H \otimes H}$ and an associated quasitriangular 
R-matrix $\mathcal{R}_{H \otimes H}$. In this way the map $\mathcal{D}(H) \cong \mathcal{M}_{R}(H) \rightarrow H \otimes_{\text {alg }} H$ induced by Mon $_{R}$ provides a homomorphism (isomorphism, if $\operatorname{mon}_{R}$ is bijective) of quasitriangular Hopf algebras, which turns out to coincide with the one already given in [RS1]. It is conjectured that after some corrections 3 the Hopf algebra structure obtained on $\mathcal{K}$ by [AFFS] should agree with these results. Furthermore, with these corrections the $\mathcal{K}$-coaction on $\mathcal{A}$ discovered by [AFFS] should also be expected to become an example from the class of universal localized $\mathcal{D}(H)$-coactions on $\mathcal{A}$ established in $[\mathrm{NSz}]$.

A more detailed account of this will be given elsewhere, where it will also be shown that, independently of the existence of $R$, the model (1.1), (1.2) with periodic boundary conditions always contains $\mathcal{D}(H)$ as a global subalgebra governing its representation theory, similarly as in [AFFS].

The Appendix concludes with an independent investigation of the multi-loop algebras $\mathcal{L}_{m}$ of [AS]. They are shown to be braided tensor products in the sense of Majid of one-loop (三 monodromy) algebras. Hence, using bosonization ideas of [M5-M8], they are naturally homomorphic (isomorphic, if $\operatorname{mon}_{R}$ is bijective) to the algebraic tensor product $H^{\otimes m}$. This explains the representation theory of $\mathcal{L}_{m}$ given in [AS].

In summary, I would once more like to emphasize that in most parts this paper should be considered as a review, unifying different notations and approaches and treating seemingly unrelated results within a common formalism. In particular, Majid's notion of braided groups is brought together with the techniques of generating matrices as advocated by the St. Petersburg school. In view of the fact that the various relations between monodromy algebras, Drinfeld doubles, braided groups and bosonization formulas seemingly have been overlooked in [AGS,AS,AFFS], this article is hoped to add a piece of clarity and also to serve a broader community working in the field.

I should point out that a rather extensive review on braided groups and braided algebras can be found in [M7] and the relation with the Drinfeld double is also reviewed in [M8], however without relating it to monodromy algebras and without using the techniques of generating matrices.

Throughout this paper all algebras are taken to be finite dimensional, but no assumptions on semi-simplicity nor on $*$-structures are made. The reader is supposed to be familiar with standard Hopf algebra theory and notation, see e.g. [Sw]. Elements of $H$ will be denoted by $a, b, c, \ldots$ and elements of $\hat{H}$ by $\varphi, \psi, \chi, \ldots$ The counit is denoted by $\varepsilon: H \rightarrow \mathbf{C}$ and the antipode by $S: H \rightarrow H$.

Acknowledgements: I would like to thank A. Alekseev and V. Schomerus for discussions on their results in [AGS,AS,AFFS]. I am also indepted to S. Majid for helpful comments and for bringing refs. [M6-M8] to my attention.

\section{Monodromy Algebras and Braided Groups}

Defining relations of so-called monodromy or loop algebras have been given in [AGS,AS], where they appear as algebras generated by the matrix elements of quantum holonomy operators around closed loops. For an earlier version based on quadratic relations see also [RS2]. In

\footnotetext{
${ }^{3}$ There were some inconsistencies in $[\mathrm{AFFS}]$, see Section 7
} 
order to establish that they are indeed well defined associative algebras we start by identifying their dual coalgebras with the braided groups introduced by Majid in [M3].

Proposition 2.1 [M3] Let $R=\sum_{i} x^{i} \otimes y^{i} \in H \otimes H$ be quasitriangular with respect to $\Delta$ and let $\Delta_{R}: H \rightarrow H \otimes H$ be a deformed coproduct given by

$$
\Delta_{R}(a)=\sum_{i, j}\left(x^{i} \otimes S\left(y^{j}\right) y^{i}\right) \Delta(a)\left(x^{j} \otimes \mathbf{1}\right), a \in H
$$

Then $\Delta_{R}$ is coassociative with counit $\varepsilon$.

Proof: Recall that quasitriangularity of $R$ means [Dr2]

$$
\begin{aligned}
(\Delta \otimes i d)(R) & =R^{13} R^{23} \\
(i d \otimes \Delta)(R) & =R^{13} R^{12} \\
R \Delta(a) R^{-1} & =\Delta_{o p}(a)
\end{aligned}
$$

This implies $(\varepsilon \otimes i d)(R)=(i d \otimes \varepsilon)(R)=\mathbf{1}$ and therefore $\varepsilon$ is a counit for $\Delta_{R}$. Moreover, (2.2)-(2.4) also imply the cocycle property [Dr2]

$$
R^{12}(\Delta \otimes i d)(R)=R^{23}(i d \otimes \Delta)(R)
$$

which is sufficient for $\Delta^{\prime}: H \rightarrow H \otimes H$

$$
\Delta^{\prime}(a):=R \Delta(a)
$$

to be coassociative. Now $\Delta^{\prime}$ obeys

$$
\Delta^{\prime}(a b)=\Delta^{\prime}(a) \Delta(b)=\Delta_{o p}(a) \Delta^{\prime}(b)
$$

for all $a, b \in H$, from which Proposition 2.1 follows by using

Lemma 2.2: Let $\Delta^{\prime}: H \rightarrow H \otimes H$ be a coassociative coproduct satisfying (2.7). Then $\Delta_{R}(a):=\sum_{j}\left(\mathbf{1} \otimes S\left(y^{j}\right)\right) \Delta^{\prime}(a)\left(x^{j} \otimes \mathbf{1}\right)$ is also coassociative.

Proof: Using (2.7) and (2.2) we compute for $a \in H$

$$
\begin{aligned}
\left(\Delta_{R} \otimes i d\right)\left(\Delta_{R}(a)\right) & =\sum_{i, j}\left[\mathbf{1} \otimes S\left(y^{i}\right) \otimes S\left(y^{j}\right)\right] \Delta^{\prime(2)}(a)\left[x_{(1)}^{j} x^{i} \otimes x_{(2)}^{j} \otimes \mathbf{1}\right] \\
& =\sum_{i, j, k}\left[\mathbf{1} \otimes S\left(y^{i}\right) \otimes S\left(y^{j} y^{k}\right)\right] \Delta^{\prime(2)}(a)\left[x^{j} x^{i} \otimes x^{k} \otimes \mathbf{1}\right]
\end{aligned}
$$

where $\Delta^{\prime}(2)=\left(\Delta^{\prime} \otimes i d\right) \circ \Delta^{\prime}=\left(i d \otimes \Delta^{\prime}\right) \circ \Delta^{\prime}$. Similarly, using (2.7) and (2.3),

$$
\begin{aligned}
\left(i d \otimes \Delta_{R}\right)\left(\Delta_{R}(a)\right) & =\sum_{i, j}\left[\mathbf{1} \otimes S\left(y_{(1)}^{i}\right) \otimes S\left(y^{k}\right) S\left(y_{(2)}^{i}\right)\right] \Delta^{\prime(2)}(a)\left[x^{i} \otimes x^{k} \otimes \mathbf{1}\right] \\
& =\sum_{i, j, k}\left[\mathbf{1} \otimes S\left(y^{i}\right) \otimes S\left(y^{k}\right) S\left(y^{j}\right)\right] \Delta^{\prime(2)}(a)\left[x^{j} x^{i} \otimes x^{k} \otimes \mathbf{1}\right] \\
& =\left(\Delta_{R} \otimes i d\right) \Delta_{R}(a)
\end{aligned}
$$

It has been remarked in equ. (32) of [M6] that the passage from $\Delta^{\prime}$ to $\Delta_{R}$ can also be viewed as a cocycle transformation in a generalized sense, i.e. by considering according to $(2.7)\left(H, \Delta^{\prime}\right)$ 
as an $(H \otimes H)$-Hopf right-module coalgebra with right action $a \triangleleft(b \otimes c):=S(b) a c$, and by acting with the $H \otimes H$-right cocycle $R_{23} \in(H \otimes H) \otimes(H \otimes H)$ (see Lemma 7.1) to transform $\Delta^{\prime} \rightarrow \Delta_{R}$

Up to a change of conventions (i.e. replacing $(H, \Delta, S, R)$ by $\left.\left(H, \Delta_{o p}, S^{-1}, R_{o p}\right)\right)$ the deformed coproduct (2.1) has first appeared in [M3], providing $H$ with the structure of what the author calls a "braided group". One should also point out here that the deformed coproducts $\Delta^{\prime}$ and $\Delta_{R}$ are no longer algebra homomorphisms. However, as has been emphasized by Majid [M3,M4], there exists a natural deformed algebra structure on $H \otimes H$, the "braided tensor product" $H \otimes_{R} H$ (see Appendix A), such that $\Delta_{R}: H_{c o p} \rightarrow H_{c o p} \otimes_{R_{o p}} H_{c o p}$ does provide an algebra homomorphism (see equ.(A.11)), where $H_{c o p}$ is the Hopf algebra $H$ with opposite coproduct $\Delta_{o p}$.

Following Theorem 4.1 of [M4], see also equ. (8) of [M5], we now pass to the algebra dual to $\left(H, \Delta_{R}\right)$.

Definition 2.3 [M4] The "braided group function algebra" $\hat{H}_{R}$ is defined to be the vector space $\hat{H}$ with multiplication $\times_{R}: \hat{H} \otimes \hat{H} \rightarrow \hat{H}$ induced by $\Delta_{R}$, i.e.

$$
\left\langle\varphi \times_{R} \psi \mid a\right\rangle:=\left\langle\varphi \otimes \psi \mid \Delta_{R}(a)\right\rangle
$$

for $\varphi, \psi \in \hat{H}$ and $a \in H$.

Note that $\Delta_{R}$ being coassociative, the new product $\times_{R}$ on $\hat{H}$ is clearly associative with unit given by $\varepsilon \in \hat{H}$. Let me now show that the Definition 2.3 coincides with the description of monodromy algebras given by [AGS,AS,AFFS] in terms of a generating matrix $\mathbf{M}$.

In what follows, $\mathcal{A}$ always denotes an arbitrary target algebra. The basic idea behind generating matrices is given by the observation that if $\hat{\mathcal{B}}$ is the algebra dual to some given finite dimensional coalgebra $\left(\mathcal{B}, \Delta_{\mathcal{B}}, \varepsilon_{\mathcal{B}}\right)$, then the relation $f(\varphi)=(\varphi \otimes i d)\left(\mathbf{F}_{\mathcal{A}}\right), \varphi \in \hat{\mathbf{B}}$, provides a one-to-one correspondence between algebra homomorphisms $f: \hat{\mathcal{B}} \rightarrow \mathcal{A}$ and "generating matrices" $\mathbf{F}_{\mathcal{A}} \in \mathcal{B} \otimes \mathcal{A}$ obeying

$$
\mathbf{F}_{\mathcal{A}}^{13} \mathbf{F}_{\mathcal{A}}^{23}=\left(\Delta_{\mathcal{B}} \otimes i d\right)\left(\mathbf{F}_{\mathcal{A}}\right) \in \mathcal{B} \otimes \mathcal{B} \otimes \mathcal{A}
$$

Clearly, $f$ is unital iff $\mathbf{F}_{\mathcal{A}}$ is unital in the sense $\left(\varepsilon_{\mathcal{B}} \otimes i d\right)\left(\mathbf{F}_{\mathcal{A}}\right)=\mathbf{1}_{\mathcal{A}}$. If $\mathcal{B}$, and therefore $\hat{\mathcal{B}}$, are bialgebras, then $\mathcal{B}$ has an antipode $S_{\mathcal{B}}$ if and only if all unital generating matrices are invertible, the relation being given by

$$
\mathbf{F}_{\mathcal{A}}^{-1}=\left(S_{\mathcal{B}} \otimes i d_{\mathcal{A}}\right)\left(\mathbf{F}_{\mathcal{A}}\right)
$$

If also $\mathcal{A}$ is a bialgebra then $f$ defines a bialgebra homomorphism if and only if in addition to $(2.9) \mathbf{F}_{\mathcal{A}}$ satisfies

$$
\mathbf{F}_{\mathcal{A}}^{12} \mathbf{F}_{\mathcal{A}}^{13}=\left(i d \otimes \Delta_{\mathcal{A}}\right)\left(\mathbf{F}_{\mathcal{A}}\right) \in \mathcal{B} \otimes \mathcal{A} \otimes \mathcal{A}
$$

Putting $\mathcal{A}=\hat{\mathcal{B}}$ and $f=i d_{\hat{\mathcal{B}}}$ one obtains $\mathbf{F} \equiv \mathbf{E}=\sum_{\nu} e_{\nu} \otimes e^{\nu} \in \mathcal{B} \otimes \hat{\mathcal{B}}$, which justifies the statement that the algebra $\hat{\mathcal{B}}$ is generated by the entries $(\varphi \otimes i d)(\mathbf{F}), \varphi \in \hat{\mathcal{B}}$, with abstract relations (2.9). Applying all this to the algebra $\hat{H}_{R}$ we get

Proposition 2.4 The relation $f(\varphi)=(\varphi \otimes i d)\left(\mathbf{M}_{\mathcal{A}}\right)$ provides a one-to-one correspondence between algebra homomorphisms $f: \hat{H}_{R} \rightarrow \mathcal{A}$ and elements $\mathbf{M}_{\mathcal{A}} \in H \otimes \mathcal{A}$ satisfying

$$
\mathbf{M}_{\mathcal{A}}^{13} R^{12} \mathbf{M}_{\mathcal{A}}^{23}=R^{12}(\Delta \otimes i d)\left(\mathbf{M}_{\mathcal{A}}\right) \in H \otimes H \otimes \mathcal{A}
$$


Proof: According to (2.8) and (2.9) we have to verify that (2.12) is equivalent to

$$
\mathbf{M}_{\mathcal{A}}^{13} \mathbf{M}_{\mathcal{A}}^{23}=\left(\Delta_{R} \otimes i d\right)\left(\mathbf{M}_{\mathcal{A}}\right)
$$

To this end we use (2.3) to get the identity

$$
\sum_{i, j} x^{i} x^{j} \otimes S\left(y^{j}\right) y^{i}=\sum_{i} x^{i} \otimes S\left(y_{(1)}^{i}\right) y_{(2)}^{i}=\mathbf{1} \otimes \mathbf{1}
$$

Hence (2.12) implies

$$
\mathbf{M}_{\mathcal{A}}^{13} \mathbf{M}_{\mathcal{A}}^{23}=\sum_{j}\left[\mathbf{1} \otimes S\left(y^{j}\right) \otimes \mathbf{1}\right] \mathbf{M}_{\mathcal{A}}^{13} R^{12} \mathbf{M}_{\mathcal{A}}^{23}\left[x^{j} \otimes \mathbf{1} \otimes \mathbf{1}\right]=\left(\Delta_{R} \otimes i d\right)\left(\mathbf{M}_{\mathcal{A}}\right)
$$

Conversely, (2.13) implies

$$
\mathbf{M}_{\mathcal{A}}^{13} R^{12} \mathbf{M}_{\mathcal{A}}^{23}=\sum_{i}\left[\mathbf{1} \otimes y^{j} \otimes \mathbf{1}\right]\left(\Delta_{R} \otimes i d\right)\left(\mathbf{M}_{\mathcal{A}}\right)\left[x^{j} \otimes \mathbf{1} \otimes \mathbf{1}\right]=R^{12}(\Delta \otimes i d)\left(\mathbf{M}_{\mathcal{A}}\right)
$$

where, similarly as above, we have used

$$
\sum_{i, j} x^{i} x^{j} \otimes y^{j} S\left(x^{i}\right)=\sum_{i} x^{i} \otimes y_{(1)}^{i} S\left(y_{(2)}^{i}\right)=\mathbf{1} \otimes \mathbf{1}
$$

Putting $\mathcal{A}=\hat{H}_{R}$ and $f=i d$, equation (2.12) coincides with the defining "monodromy relations" given in [AFFS]. The convention of [AGS,AS] differs by a multiplication of $\mathbf{M}_{\mathcal{A}}$ with $\left(\kappa^{-1} \otimes \mathbf{1}_{\mathcal{A}}\right)$, where $\kappa \in H$ is a certain central square root of a ribbon element associated with $R$.

Hence, from now on we will call $\times_{R}$ the monodromy product on $\hat{H}$ and $\hat{H}_{R}$ the monodromy algebra associated with $(H, R)$.

\section{Gauged Monodromy Algebras}

Following Majid [M4,M5] we now provide a coadjoint action of $H$ on $\hat{H}_{R}$ which becomes a Hopf module action with respect to the monodromy product $\times_{R}$. Again we start with the dual point of view by first recalling that $\Delta_{R}$ is an $H$-module map with respect to the right adjoint action of $H$ on itself (this is a basic ingredient in Majid's construction of braided groups by what he calls "transmutation").

Lemma 3.1 For $a, b \in H$ we have

$$
\Delta_{R}\left(S\left(a_{(1)}\right) b a_{(2)}\right)=\left[S\left(a_{(1)}\right) \otimes S\left(a_{(3)}\right)\right] \Delta_{R}(b)\left[a_{(2)} \otimes a_{(4)}\right]
$$

Proof: By straight forward verification, using (2.4), (2.7) and the identity $\Delta \circ S=(S \otimes S) \circ \Delta_{o p}$.

Corollary 3.2 Let $\triangleright: H \otimes \hat{H}_{R} \ni a \otimes \varphi \mapsto a \triangleright \varphi \in \hat{H}_{R}$ be given by

$$
\langle a \triangleright \varphi \mid b\rangle:=\left\langle\varphi \mid S\left(a_{(1)}\right) b a_{(2)}\right\rangle
$$

where $a, b \in H$ and $\varphi \in \hat{H}_{R}$. Then $\triangleright$ defines a Hopf module left action of $H$ on $\hat{H}_{R}$. 
Proof: The identities $a \triangleright(b \triangleright \varphi)=(a b) \triangleright \varphi, \mathbf{1} \triangleright \varphi=\varphi$ and $a \triangleright \varepsilon=\varepsilon(a) \varepsilon$ are obvious. The Hopf module property

$$
a \triangleright\left(\varphi \times_{R} \psi\right)=\left(a_{(1)} \triangleright \varphi\right) \times_{R}\left(a_{(2)} \triangleright \psi\right)
$$

follows by pairing both sides with $b \in H$ and using (2.8) and (3.1).

Definition 3.3 The "gauged monodromy algebra" $\mathcal{M}_{R}(H)$ is defined to be the crossed product

$$
\mathcal{M}_{R}(H):=\hat{H}_{R} \rtimes H
$$

Thus, recalling the definition of a crossed product, $\mathcal{M}_{R}(H)$ is the linear space $\hat{H} \otimes H$ with associative multiplication

$$
(\varphi \otimes a)(\psi \otimes b):=\left(\varphi \times_{R}\left(a_{(1)} \triangleright \psi\right) \otimes a_{(2)} b\right),
$$

where $\varphi, \psi \in \hat{H}$ and $a, b \in H$. To distinguish the new algebraic structure we denote the images of $\hat{H}_{R}$ and $H$ in $\mathcal{M}_{R}(H)$ by $i_{M}(a) \equiv \varepsilon \otimes a, a \in H$, and $M(\varphi) \equiv \varphi \otimes \mathbf{1}_{H}, \varphi \in \hat{H}$. We also denote $\mathbf{M}:=\sum e_{\nu} \otimes M\left(e^{\nu}\right) \in H \otimes \mathcal{M}_{R}(H)$.

The crossed product $\mathcal{M}_{R}(H)$ has first appeared in [M2,M5]. Using the generating-matrix formalism we now show that $\mathcal{M}_{R}(H)$ coincides with the algebra $\mathcal{K}$ given in Definition 5 of [AFFS] (see also [AGS,AS]) as the subalgebra generated inside a current algebra by a monodromy operator $\mathbf{M}$ together with the algebra of gauge transformations sitting at its initial (三 end) point.

Proposition 3.4 Let $f: H \rightarrow \mathcal{A}$ be an algebra homomorphism. Then the relation

$$
f_{M}(\varphi \otimes a):=(\varphi \otimes i d)\left(\mathbf{M}_{\mathcal{A}}\right) f(a)
$$

provides a one-to-one correspondence between algebra homomorphisms $f_{M}: \mathcal{M}_{R}(H) \rightarrow \mathcal{A}$ extending $f$ and elements $\mathbf{M}_{\mathcal{A}} \in H \otimes \mathcal{A}$ satisfying (2.12) together with $(\varepsilon \otimes i d)\left(\mathbf{M}_{\mathcal{A}}\right)=f\left(\mathbf{1}_{H}\right)$ and

$$
\left[a_{(1)} \otimes f\left(a_{(2)}\right)\right] \mathbf{M}_{\mathcal{A}}=\mathbf{M}_{\mathcal{A}}\left[a_{(1)} \otimes f\left(a_{(2)}\right)\right], \quad \forall a \in H .
$$

Proof: Since $\mathbf{1}_{\mathcal{M}_{R}(H)}=\varepsilon \otimes \mathbf{1}_{H}$, the condition $(\varepsilon \otimes i d)\left(\mathbf{M}_{\mathcal{A}}\right)=f\left(\mathbf{1}_{H}\right)$ is equivalent to $f_{M}\left(\mathbf{1}_{\mathcal{M}_{R}(H)}\right)=f\left(\mathbf{1}_{H}\right)$. We are left to show that (3.5) holds if and only if $f_{M}$ respects (3.4), or equivalently, if and only if

$$
f\left(a_{(1)}\right)\left(\varphi \otimes i d_{\mathcal{A}}\right)\left(\mathbf{M}_{\mathcal{A}}\right) f\left(S\left(a_{(2)}\right)\right)=((a \triangleright \varphi) \otimes i d)\left(\mathbf{M}_{\mathcal{A}}\right)
$$

for all $\varphi \in \hat{H}$ and all $a \in H$. Now (3.6) is equivalent to

$$
\left(\mathbf{1}_{H} \otimes f\left(a_{(1)}\right)\right) \mathbf{M}_{\mathcal{A}}\left(\mathbf{1}_{H} \otimes f\left(S\left(a_{(2)}\right)\right)=\left(S\left(a_{(1)}\right) \otimes \mathbf{1}_{\mathcal{A}}\right)\left(\mathbf{M}_{\mathcal{A}}\right)\left(a_{(2)} \otimes \mathbf{1}_{\mathcal{A}}\right)\right.
$$

which further implies

$$
\begin{aligned}
{\left[a_{(1)} \otimes f\left(a_{(2)}\right)\right] \mathcal{M}_{\mathcal{A}} } & =\left[a_{(1)} \otimes f\left(a_{(2)}\right)\right] \mathbf{M}_{\mathcal{A}}\left[\mathbf{1}_{H} \otimes f\left(S\left(a_{(3)}\right) a_{(4)}\right)\right] \\
& =\left[a_{(1)} S\left(a_{(2)}\right) \otimes \mathbf{1}_{\mathcal{A}}\right] \mathbf{M}_{\mathcal{A}}\left[a_{(3)} \otimes f\left(a_{(4)}\right)\right] \\
& =\mathbf{M}_{\mathcal{A}}\left[a_{(2)} \otimes f\left(a_{(2)}\right)\right]
\end{aligned}
$$

and therefore (3.5). Conversely, given (3.5) we conclude

$$
\begin{aligned}
{\left[\mathbf{1}_{H} \otimes f\left(a_{(1)}\right)\right] \mathbf{M}_{\mathcal{A}}\left[\mathbf{1}_{H} \otimes f\left(S\left(a_{(2)}\right)\right)\right] } & =\left[S\left(a_{(1)}\right) a_{(2)} \otimes f\left(a_{(3)}\right)\right] \mathbf{M}_{\mathcal{A}}\left[\mathbf{1}_{H} \otimes f\left(S\left(a_{(4)}\right)\right)\right] \\
& =\left[S\left(a_{(1)}\right) \otimes \mathbf{1}_{\mathcal{A}}\right] \mathbf{M}_{\mathcal{A}}\left[a_{(2)} \otimes f\left(a_{(3)} S\left(a_{(4)}\right)\right)\right] \\
& =\left[S\left(a_{(1)}\right) \otimes \mathbf{1}_{\mathcal{A}}\right] \mathbf{M}_{\mathcal{A}}\left[a_{(2)} \otimes \mathbf{1}_{\mathcal{A}}\right]
\end{aligned}
$$

and therefore (3.7). 


\section{Left and Right Monodromies}

Here we review the observation of $\left[\right.$ AFFS] on a right monodromy algebra $\hat{H}_{R}^{r} \subset \mathcal{M}_{R}(H)$ commuting with $\hat{H}_{R}^{\ell} \equiv \hat{H}_{R} \subset \mathcal{M}_{R}(H)$. First we define $\hat{H}_{R}^{r}$ abstractly as in Definition 2.3, where we replace $(H, \Delta, R, S)$ by $\left(H, \Delta_{o p}, R_{o p}, S^{-1}\right)$, i.e. the quasitriangular Hopf algebra $H$ with opposite coproduct. Similarly, to define a left Hopf module action of $H_{c o p} \equiv\left(H, \Delta_{o p}\right)$ on $\hat{H}_{R}^{r}$ we replace $(3.2)$ by

$$
\left\langle a \triangleright^{\prime} \varphi \mid b\right\rangle:=\left\langle\varphi \mid S^{-1}\left(a_{(2)}\right) b a_{(1)}\right\rangle
$$

leading to a crossed product

$$
\mathcal{M}_{R}^{r}(H):=\hat{H}_{R}^{r} \rtimes H_{c o p}
$$

for which Propositions 2.4 and 3.4 hold analogously, with $(\Delta, R)$ replaced by $\left(\Delta_{o p}, R_{o p}\right)$. Putting in particular $\mathcal{A}=\mathcal{M}_{R}^{\ell}(H) \equiv \mathcal{M}_{R}(H)$ we now get an isomorphism $\mathcal{M}_{R}^{r}(H) \rightarrow \mathcal{M}_{R}^{\ell}(H)$ restricting to the identity on $H$ by choosing in Proposition $3.4 \mathbf{M}_{\mathcal{A}}=\mathbf{M}^{r}$,

$$
\mathbf{M}^{r}:=\mathbf{R}\left(\mathbf{M}^{\ell}\right)^{-1} \mathbf{R}_{o p} \in H \otimes \mathcal{M}_{R}^{\ell}(H)
$$

where $\mathbf{M}^{\ell} \equiv \mathbf{M}:=\sum e_{\nu} \otimes M\left(e^{\nu}\right) \in H \otimes \mathcal{M}_{R}^{\ell}(H)$ and where $\mathbf{R}_{(o p)}:=\left(i d \otimes i_{M}\right)\left(R_{(o p)}\right) \in$ $H \otimes \mathcal{M}_{R}^{\ell}(H)$. This is the formula of [AFFS], which is proven by equ. (4.6) below. Note however that for (4.3) to be well defined we first have to assure that $\mathbf{M}^{\ell}$ is invertible in $H \otimes \mathcal{M}_{R}^{\ell}(H)$.

Proposition 4.1 i) For any target algebra $\mathcal{A}$ let $f: H \rightarrow \mathcal{A}$ be a unital homomorphism and let $\mathbf{M}_{\mathcal{A}}^{\ell} \equiv \mathbf{M}_{\mathcal{A}} \in H \otimes \mathcal{A}$ be unital and satisfy (2.12) and (3.5). Then

$$
\mathbf{M}_{\mathcal{A}}^{-1}=(S \otimes i d)\left((i d \otimes f)\left(R_{o p}^{-1}\right) \mathbf{M}_{\mathcal{A}}\right)(i d \otimes f)\left(R_{o p}^{-1}\right) \in H \otimes \mathcal{A}
$$

ii) Putting $\mathbf{M}_{\mathcal{A}}^{r}:=(i d \otimes f)(R)\left(\mathbf{M}_{\mathcal{A}}^{\ell}\right)^{-1}(i d \otimes f)\left(R_{o p}\right)$ we have

$$
\begin{aligned}
{\left[a_{(2)} \otimes f\left(a_{(1)}\right)\right] \mathbf{M}_{\mathcal{A}}^{r} } & =\mathbf{M}_{\mathcal{A}}^{r}\left[a_{(2)} \otimes f\left(a_{(1)}\right)\right] \\
\left(\mathbf{M}_{\mathcal{A}}^{r}\right)^{13} R^{21}\left(\mathbf{M}_{\mathcal{A}}^{r}\right)^{23} & =R^{21}\left(\Delta_{o p} \otimes i d\right)\left(\mathbf{M}_{\mathcal{A}}^{r}\right) \\
\left(\mathbf{M}_{\mathcal{A}}^{\ell}\right)^{13}\left(\mathbf{M}_{\mathcal{A}}^{r}\right)^{23} & =\left(\mathbf{M}_{\mathcal{A}}^{r}\right)^{23}\left(\mathbf{M}_{\mathcal{A}}^{\ell}\right)^{13}
\end{aligned}
$$

Proof: i) Putting

$$
\mathbf{D}_{\mathcal{A}}:=(i d \otimes f)\left(R_{o p}^{-1}\right) \mathbf{M}_{\mathcal{A}} \in H \otimes \mathcal{A}
$$

and using (2.5) we have in $H \otimes H \otimes \mathcal{A}$

$$
R^{12}(\Delta \otimes i d)\left(\mathbf{M}_{\mathcal{A}}\right)=[(R \otimes \mathbf{1})(\Delta \otimes i d)(R)]^{312}(\Delta \otimes i d)\left(\mathbf{D}_{\mathcal{A}}\right)
$$

On the other hand (2.2) and (2.4) imply

$$
\begin{aligned}
\mathbf{M}_{\mathcal{A}}^{13} R^{12} \mathbf{M}_{\mathcal{A}}^{23} & =\mathbf{M}_{\mathcal{A}}^{13}(\Delta \otimes i d)(R)^{132} \mathbf{D}_{\mathcal{A}}^{23} \\
& =\left[(\Delta \otimes i d)(R)\left(R_{o p} \otimes \mathbf{1}\right)\right]^{132} \mathbf{D}_{\mathcal{A}}^{13} \mathbf{D}_{\mathcal{A}}^{23} \\
& =[(R \otimes \mathbf{1})(\Delta \otimes i d)(R)]^{312} \mathbf{D}_{\mathcal{A}}^{13} \mathbf{D}_{\mathcal{A}}^{23}
\end{aligned}
$$

where in the second line we have used (3.5) and where by a convenient abuse of notation we have dropped the symbol $f$. Hence comparing (4.9) and (4.10) and using (2.12) we conclude

$$
\mathbf{D}_{\mathcal{A}}^{13} \mathbf{D}_{\mathcal{A}}^{23}=\left(\Delta \otimes i d_{\mathcal{A}}\right)\left(\mathbf{D}_{\mathcal{A}}\right)
$$


Now $\mathbf{D}_{\mathcal{A}}$ is unital since $f$ and $\mathbf{M}_{\mathcal{A}}$ are unital and, therefore, (4.11) implies by (2.10)

$$
\mathbf{D}_{\mathcal{A}}^{-1}=\left(S \otimes i d_{\mathcal{A}}\right)\left(\mathbf{D}_{\mathcal{A}}\right)
$$

from which (4.4) follows.

ii) Equ.(4.5) immediately follows from (2.4) and (3.5). To prove (4.6) we compute, omitting the symbol $f$,

$$
\begin{aligned}
\left(\mathbf{M}_{\mathcal{A}}^{r}\right)^{23} R^{12}\left(\mathbf{M}_{\mathcal{A}}^{r}\right)^{13} & =R^{23}\left(\mathbf{M}_{\mathcal{A}}^{-1}\right)^{23} R^{32} R^{12} R^{13}\left(\mathbf{M}_{\mathcal{A}}^{-1}\right)^{13} R^{31} \\
& =R^{23}\left(\mathcal{M}_{\mathcal{A}}^{-1}\right)^{23}(i d \otimes \Delta)(R)\left(R^{-1}\right)^{12}(\Delta \otimes i d)(R)^{132}\left(\mathbf{M}_{\mathcal{A}}^{-1}\right)^{13} R^{31} \\
& =R^{12}(\Delta \otimes i d)(R)\left[\left(\mathbf{M}_{\mathcal{A}}^{-1}\right)^{23}\left(R^{-1}\right)^{12}\left(\mathbf{M}_{\mathcal{A}}^{-1}\right)^{13}\right](\Delta \otimes i d)(R)^{132} R^{31} \\
& =R^{12}(\Delta \otimes i d)\left(\mathbf{M}_{\mathcal{A}}^{r} R_{o p}^{-1}\right)\left(R^{12}\right)^{-1} R^{12} R^{32} R^{31} \\
& =R^{12}(\Delta \otimes i d)\left(\mathbf{M}_{\mathcal{A}}^{r}\right)
\end{aligned}
$$

Here we have used (2.2)-(2.4) in the second line, (3.5) and (2.5) in the third line, (2.2), (4.3) and the inverse of (2.12) in the fourth line and again (2.3) in the last line. Applying the permutation $(12) \rightarrow(21)$ to both sides this proves (4.6). Finally, to prove (4.7) we put $\mathbf{M} \equiv M_{\mathcal{A}}^{\ell}$ and $\Omega \equiv\left(M_{\mathcal{A}}^{r}\right)^{-1}$ and compute

$$
\begin{aligned}
\mathbf{M}^{13} \Omega^{23} & =\mathbf{M}^{13}\left(R^{12} R^{32}\right)^{-1} R^{12} \mathbf{M}^{23}\left(R^{23}\right)^{-1} \\
& =(\Delta \otimes i d)\left(R^{-1}\right)^{132} R^{12}(\Delta \otimes i d)(\mathbf{M})\left(R^{23}\right)^{-1} \\
& =\left(R^{32}\right)^{-1}(\Delta \otimes i d)(\mathbf{M})\left(R^{23}\right)^{-1}
\end{aligned}
$$

where in the second line we have used (2.2), (3.5) and (2.12), and in the third line again (2.2). Similarly one obtains

$$
\begin{aligned}
\Omega^{23} \mathbf{M}^{13} & =\left(R^{32}\right)^{-1} \mathbf{M}^{23} R^{21}\left(R^{23} R^{21}\right)^{-1} \mathbf{M}^{13} \\
& =\left(R^{32}\right)^{-1} R^{21}\left(\Delta_{o p} \otimes i d\right)(\mathbf{M})(i d \otimes \Delta)\left(R^{-1}\right)^{213} \\
& =\left(R^{32}\right)^{-1} R^{21}\left(\Delta_{o p} \otimes i d\right)(\mathbf{M})\left(R^{21}\right)^{-1}\left(R^{23}\right)^{-1} \\
& =M^{13} \Omega^{23}
\end{aligned}
$$

which proves (4.7).

Note that (4.7) implies that the image of $\hat{H}_{R}^{r}$ in $\mathcal{M}_{R}^{\ell}(H)$ indeed commutes with $\hat{H}_{R}^{\ell}$. Part ii) of Proposition 4.1 has been taken over from [AFFS].

\section{The Drinfeld Double}

The Drinfeld double $\mathcal{D}(H)$ (also called quantum double) has been introduced in [Dr1] and is meanwhile well understood as a double crossed product of $H$ with $\hat{H}$ [M1,M8], see also [RS1,K].

Definition 5.1 The Drinfeld double $\mathcal{D}(H)$ over a finite dimensional Hopf algebra $H$ is the linear space $\hat{H} \otimes H$ with multiplication given for $a, b \in H$ and $\varphi, \psi \in \hat{H}$ by

$$
(\varphi \otimes a)(\psi \otimes b):=\left(\varphi \psi_{(2)} \otimes a_{(2)} b\right)\left\langle a_{(1)} \mid \psi_{(3)}\right\rangle\left\langle\psi_{(1)} \mid S^{-1}\left(a_{(3)}\right)\right\rangle
$$

Putting $i_{D}(a):=\left(\mathbf{1}_{\hat{H}} \otimes a\right)$ and $D(\varphi):=\left(\varphi \otimes \mathbf{1}_{H}\right)$ one can rewrite this equivalently as [NSz]

$$
\begin{aligned}
i_{D}(a) i_{D}(b) & =i_{D}(a b) \\
D(\varphi) D(\psi) & =D(\varphi \psi) \\
D\left(\varphi_{(1)}\right)\left\langle\varphi_{(2)} \mid a_{(1)}\right\rangle i_{D}\left(a_{(2)}\right) & =i_{D}\left(a_{(1)}\right)\left\langle a_{(2)} \mid \varphi_{(1)}\right\rangle D\left(\varphi_{(2)}\right)
\end{aligned}
$$


Hence, as an algebra $\mathcal{D}(H)=\mathcal{D}(\hat{H})$. Based on a more general setting given by [Ra] it has first been noticed in [M2] (see also Proposition 4.1 of [M5]) that $\mathcal{D}(H)$ is in fact isomorphic to $\mathcal{M}_{R}(H)$ for all quasitriangular $R \in H \otimes H$.

Using our formalism of generating matrices let me now demonstrate that this result reduces to a Corollary of the calculation leading to (4.11). First we need an analogue of Proposition 3.4. Introducing $\mathbf{D}:=\sum_{\nu} e_{\nu} \otimes D\left(e^{\nu}\right) \in H \otimes \mathcal{D}(H)$ we note that (5.3) is equivalent to

$$
\mathbf{D}^{13} \mathbf{D}^{23}=(\Delta \otimes i d)(\mathbf{D})
$$

and (5.4) is equivalent to

$$
\mathbf{D}\left[a_{(1)} \otimes i_{D}\left(a_{(2)}\right)\right]=\left[a_{(2)} \otimes i_{D}\left(a_{(1)}\right)\right] \mathbf{D}
$$

More generally this leads to

Lemma 5.2 Let $f: H \rightarrow \mathcal{A}$ be an algebra homomorphism. Then the relation

$$
f_{D}(\varphi \otimes a)=\left(\varphi \otimes i d_{\mathcal{A}}\right)\left(\mathbf{D}_{\mathcal{A}}\right) f(a), a \in H, \varphi \in \hat{H}
$$

provides a one-to-one correspondence between algebra homomorphisms $f_{D}: \mathcal{D}(H) \rightarrow \mathcal{A}$ extending $f$ and elements $\mathbf{D}_{\mathcal{A}} \in H \otimes \mathcal{A}$ obeying $(\varepsilon \otimes i d)\left(\mathbf{D}_{\mathcal{A}}\right)=f\left(\mathbf{1}_{H}\right)$ and

$$
\begin{aligned}
\mathbf{D}_{\mathcal{A}}^{13} \mathbf{D}_{\mathcal{A}}^{23} & =(\Delta \otimes i d)\left(\mathbf{D}_{\mathcal{A}}\right) \\
\mathbf{D}_{\mathcal{A}}\left[a_{(1)} \otimes f\left(a_{(2)}\right)\right] & =\left[a_{(2)} \otimes f\left(a_{(1)}\right)\right] \mathbf{D}_{\mathcal{A}}, \quad a \in H
\end{aligned}
$$

Proof: First, by (2.9) the $\mathbf{D}_{\mathcal{A}}$ 's satisfying $(5.5)$ are in one-to-one correspondence with homomorphisms $\hat{H} \rightarrow \mathcal{A}$. Since $\mathbf{1}_{\mathcal{D}(H)}=i_{D}\left(\mathbf{1}_{H}\right)$, the condition $(\varepsilon \otimes i d)\left(\mathbf{D}_{\mathcal{A}}\right)=f\left(\mathbf{1}_{H}\right)$ is equivalent to $f_{D}\left(\mathbf{1}_{\mathcal{D}(H)}\right)=f\left(\mathbf{1}_{H}\right)$. We are left to show that $f_{D}$ respects (5.4) if and only if $\mathbf{D}_{\mathcal{A}}$ obeys (5.6), which may immediately be realized by applying $\left(\varphi \otimes i d_{\mathcal{A}}\right)$ to 5.6 ).

Inspired by (4.8) and (4.11) we now put $\mathcal{A}=\mathcal{M}_{R}(H), f=i_{M}: H \rightarrow \mathcal{M}_{R}(H)$ the canonical embedding and

$$
\mathbf{D}_{M}:=\mathbf{R}_{o p}^{-1} \mathbf{M} \in H \otimes \mathcal{M}_{R}(H)
$$

where $\mathbf{R}_{o p}:=\left(i d \otimes i_{M}\right)\left(R_{o p}\right) \in H \otimes \mathcal{M}_{R}(H)$ and $\mathbf{M}=\sum e_{\nu} \otimes M\left(e^{\nu}\right)$ as before. Then we have

Corollary $5.3[\mathrm{M} 2, \mathrm{M} 5]$ The element $\mathbf{D}_{M}$ (5.7) defines an algebra isomorphism $\lambda_{R}: \mathcal{D}(H) \rightarrow$ $\mathcal{M}_{R}(H)$ restricting to the identity on $H$ by putting

$$
\lambda_{R}(\varphi \otimes a):=(\varphi \otimes i d)\left(\mathbf{D}_{M}\right) i_{M}(a) .
$$

Proof: We apply Lemma 5.2. Equ. (5.5) has already been verified in (4.11). Equ. (5.6) follows from (3.5) and (2.4). Finally, $\lambda_{R}$ is invertible with $\lambda_{R}^{-1}$ given according to Proposition 3.4 by

$$
\mathbf{M}_{D}:=\left(i d \otimes i_{D}\right)\left(R_{o p}\right) \mathbf{D} \in H \otimes \mathcal{D}(H)
$$

Denoting $\mathbf{M}_{D}^{\ell} \equiv \mathbf{M}_{D}$ and looking at (4.3) we also get an immediate formula for a right monodromy $\mathbf{M}_{D}^{r} \in H \otimes \mathcal{D}(H)$

$$
\mathbf{M}_{D}^{r}:=\left(i d \otimes i_{D}\right)(R) \mathbf{D}^{-1}
$$

Equ. (4.7) then implies that the subalgebras $\left\langle\hat{H} \otimes i d \mid \mathbf{M}_{D}^{r}\right\rangle \cong \hat{H}_{R}^{r}$ and $\left\langle\hat{H} \otimes i d \mid \mathbf{M}_{D}^{\ell}\right\rangle \cong \hat{H}_{R}^{\ell}$ commute inside $\mathcal{D}(H)$. In the next section we will review the factorization condition of [RS1] guaranteeing $\mathcal{M}_{R}(H) \equiv \mathcal{D}(H) \cong \hat{H}_{R}^{\ell} \otimes \hat{H}_{R}^{r}$. 


\section{The Monodromy Homomorphism}

Following Propositions 2.1 and 2.2 of [M5] we now provide what may be called the monodromy homomorphism $\hat{H}_{R} \rightarrow H$, which as a linear map has already been discussed in [RS1].

Proposition 6.1 [M5] Let $\operatorname{mon}_{R}: \hat{H} \rightarrow H$ be the linear map given by

$$
\operatorname{mon}_{R}(\varphi):=(\varphi \otimes i d)\left(R_{o p} R\right), \quad \varphi \in \hat{H}
$$

Then $\operatorname{mon}_{R}$ provides an algebra homomorphism $\operatorname{mon}_{R}: \hat{H}_{R} \rightarrow H$ satisfying for $a \in H$ and $\varphi \in \hat{H}$

$$
\operatorname{mon}_{R}(a \triangleright \varphi)=a_{(1)} \operatorname{mon}_{R}(\varphi) S\left(a_{(2)}\right)
$$

Proof: Putting $\mathcal{A}=H$ in Proposition 2.4 we have to check

$$
\left(R^{31} R^{13}\right) R^{12}\left(R^{32} R^{23}\right)=R^{12}(\Delta \otimes i d)\left(R_{o p} R\right)
$$

which is straight forward from the quasitriangularity of $R$. To prove (6.1) we use the definition (3.2) to compute

$$
\begin{aligned}
\operatorname{mon}_{R}(a \triangleright \varphi) & =\left\langle\varphi \otimes i d \mid\left(S\left(a_{(1)}\right) \otimes \mathbf{1}\right) R_{o p} R\left(a_{(2)} \otimes \mathbf{1}\right)\right\rangle \\
& =\left\langle\varphi \otimes i d \mid\left(S\left(a_{(1)}\right) \otimes \mathbf{1}\right) R_{o p} R\left(a_{(2)} \otimes a_{(3)} S\left(a_{(4)}\right)\right)\right\rangle \\
& =\left\langle\varphi \otimes i d \mid\left(S\left(a_{(1)}\right) a_{(2)} \otimes a_{(3)}\right) R_{o p} R\left(\mathbf{1} \otimes S\left(a_{(4)}\right)\right)\right\rangle \\
& =\left\langle\varphi \otimes i d \mid\left(\mathbf{1} \otimes a_{(1)}\right) R_{o p} R\left(\mathbf{1} \otimes S\left(a_{(2)}\right)\right)\right\rangle \\
& =a_{(1)} \operatorname{mon}_{R}(\varphi) S\left(a_{(2)}\right)
\end{aligned}
$$

where in the third line we have used that $R_{o p} R$ commutes with $\Delta(H)$.

Proposition 6.1 has implicitely been used in [AS] when studying representations of the monodromy algebra $\hat{H}_{R}$ in terms of representations of $H$.

Next, since $(\operatorname{Ad} a) b:=a_{(1)} b S\left(a_{(2)}\right), a, b \in H$, defines a Hopf module action of $H$ on itself, Proposition 6.1 immediately implies that $\operatorname{mon}_{R}$ extends to a homomorphism Mon $_{R}$ of the associated crossed products.

Corollary 6.2 [M5] The map $\operatorname{Mon}_{R}: \hat{H} \otimes H \rightarrow H \otimes H$

$$
\operatorname{Mon}_{R}(\varphi \otimes a):=\operatorname{mon}_{R}(\varphi) \otimes a
$$

provides a homomorphism of algebras $\operatorname{Mon}_{R}: \mathcal{M}_{R}(H) \rightarrow H \rtimes_{A d} H$.

We now note a simple "bosonization formula" f showing that as an algebra the crossed product $H \rtimes_{A d} H$ is in fact isomorphic to $H \otimes_{\text {alg }} H$.

Lemma 6.3 Let $U: H \otimes H \rightarrow H \otimes H$ be given by $U(a \otimes b):=a b_{(1)} \otimes b_{(2)}$. Then $U$ defines an algebra isomorphism $H \rtimes_{A d} H \rightarrow H \otimes_{\text {alg }} H$.

Proof: $U$ is invertible with $U^{-1}(a \otimes b)=a S\left(b_{(1)}\right) \otimes b_{(2)}$. Now the multiplication in $H \rtimes_{A d} H$ is given by

$$
\left(a \otimes_{A d} b\right)\left(c \otimes_{A d} d\right)=\left(a\left(\operatorname{Ad} b_{(1)}\right) c \otimes_{A d} b_{(2)} d\right) .
$$

\footnotetext{
${ }^{4}$ This terminology is not meant to indicate physical interpretations here. It just memorizes the fact, that the two non-commuting copies of $H$ generating $H \rtimes_{A d} H$ are traded for the two commuting copies $U^{-1}\left(H \otimes_{\text {alg }} \mathbf{1}_{H}\right)$ and $U^{-1}\left(\mathbf{1}_{H} \otimes_{\text {alg }} H\right)$.
} 
Applying $U$ to the r.h.s of (6.4) gives

$$
U\left(a b_{(1)} c S\left(b_{(2)}\right) \otimes_{A d} b_{(3)} d\right)=a b_{(1)} c d_{(1)} \otimes b_{(2)} d_{(2)}=\left[U\left(a \otimes_{A d} b\right)\right]\left[U\left(c \otimes_{A d} d\right)\right]
$$

where the last product is taken in $H \otimes_{\text {alg }} H$.

Note that Lemma 6.3 immediately generalizes to any crossed product by inner actions. Putting $\pi_{R}:=U \circ \operatorname{Mon}_{R}$ we now arrive at

Theorem 6.4 Let $\mathbf{M}^{\ell} \equiv \mathbf{M}$ and $\mathbf{M}^{r}=\mathbf{R}\left(\mathbf{M}^{\ell}\right)^{-1} \mathbf{R}_{o p}$ as in (4.3) and consider the conditions i)-iii) on an algebra homomorphism $\pi_{R}: \mathcal{M}_{R}(H) \rightarrow H \otimes_{\text {alg }} H$

$$
\begin{aligned}
\pi_{R} \circ i_{M} & =\Delta_{H} \\
i i)\left(i d_{H} \otimes \pi_{R}\right)\left(\mathbf{M}^{\ell}\right) & =R^{21} R^{12} \\
\text { iii) }\left(i d_{H} \otimes \pi_{R}\right)\left(\mathbf{M}^{r}\right) & =R^{13} R^{31}
\end{aligned}
$$

Then given i) properties ii) and iii) are equivalent with unique solution $\pi_{R}=U \circ \operatorname{Mon}_{R}$. In this case $\pi_{R}$ is an isomorphism if and only if $\operatorname{mon}_{R}$ is bijective implying $\pi_{R}^{-1}\left(H \otimes_{\text {alg }} \mathbf{1}\right)=$ $\hat{H}_{R}^{\ell}, \pi_{R}^{-1}\left(\mathbf{1} \otimes_{\text {alg }} H\right)=\hat{H}_{R}^{r}$, and therefore $\mathcal{M}_{R}(H)=\hat{H}_{R}^{\ell} \otimes_{\text {alg }} \hat{H}_{R}^{r}$.

Proof: According to (6.3), (6.2) and Proposition 3.4 $\operatorname{Mon}_{R}$ is the unique homomorphism $\mathcal{M}_{R}(H) \rightarrow H \rtimes_{A d} H$ satisfying $\operatorname{Mon}_{R} \circ i_{M}=\mathbf{1}_{H} \otimes i d_{H}$ and $\left(i d_{H} \otimes \operatorname{Mon}_{R}\right)\left(\mathbf{M}^{\ell}\right)=R^{21} R^{12} \in$ $H \otimes\left(H \otimes \mathbf{1}_{H}\right) \subset H \otimes\left(H \rtimes_{A d} H\right)$. Hence $\pi_{R}=U \circ \operatorname{Mon}_{R}$ is the unique homomorphism $\mathcal{M}_{R}(H) \rightarrow H \otimes_{\text {alg }} H$ satisfying i) and ii). Moreover, given i) conditions ii) and iii) are equivalent, since by $(2.2)-(2.4)$

$$
(i d \otimes \Delta)(R)\left(R^{21} R^{12}\right)^{-1}(i d \otimes \Delta)\left(R_{o p}\right)=R^{13} R^{31}
$$

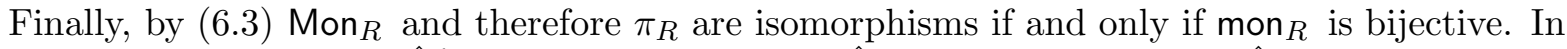
this case ii) implies $\pi_{R}\left(\hat{H}_{R}^{\ell}\right)=H \otimes_{\text {alg }} \mathbf{1}_{H}$ and $\pi_{R}\left(\hat{H}_{R}^{r}\right)=\mathbf{1}_{H} \otimes_{\text {alg }} \operatorname{mon}_{R_{o p}}(\hat{H})$, where

$$
\operatorname{mon}_{R_{o p}}(\varphi):=(\varphi \otimes i d)\left(R R_{o p}\right) \equiv(i d \otimes \varphi)\left(R_{o p} R\right)
$$

provides the monodromy homomorphism $\hat{H}_{R}^{r} \rightarrow H$. Since for $\varphi, \psi \in \hat{H}$

$$
\left\langle\varphi \mid \operatorname{mon}_{R_{o p}}(\psi)\right\rangle=\left\langle\operatorname{mon}_{R}(\varphi) \mid \psi\right\rangle
$$

we conclude that $\operatorname{mon}_{R_{o p}}$ is bijective iff $\operatorname{mon}_{R}$ is bijective, in which case $\pi_{R}\left(\hat{H}_{R}^{r}\right)=\mathbf{1}_{H} \otimes_{\text {alg }} H$.

Theorem 6.4 is the true reason underlying the observation of [AFFS] on the equivalence of the categories $\operatorname{Rep}\left(\mathcal{M}_{R}(H)\right)$ and $\operatorname{Rep}\left(H \otimes_{\text {alg }} H\right)$ provided the map $\operatorname{mon}_{R}$ is invertible. In fact, all representations of $\mathcal{M}_{R}(H)$ given in [AFFS] are of the form $\tau \circ \pi_{R}, \tau \in \operatorname{Rep}\left(H \otimes_{\text {alg }} H\right)$. Note, however, that in our approach no assumptions on semi-simplicity have been made.

\section{The Hopf Algebra Structure}

In this section we use the isomorphism $\lambda_{R}: \mathcal{D}(H) \rightarrow \mathcal{M}_{R}(H)$ of Corollary 5.3 to induce a homomorphism $\Lambda_{R}:=\pi_{R} \circ \lambda_{R}: \mathcal{D}(H) \rightarrow H \otimes_{\text {alg }} H$. We then show that up to cocycle equivalence $\Lambda_{R}$ provides a homomorphism of quasitriangular Hopf algebras, which becomes an isomorphism if and only if $\operatorname{mon}_{R}$ is bijectiv. In this way we recover the result of [RS1], where 
the invertibility property of $\operatorname{mon}_{R}$ has been called factorizability. The results of this section are also reviewed in Chapter 7 of [M8], however without using our formalism of generating matrices.

First we recall [Dr1] that $\mathcal{D}(H)$ always is a quasitriangular Hopf algebra with coproduct $\Delta_{D}$, antipode $S_{D}$ and R-matrix $R_{D}$ given for $a \in H$ and $\varphi \in \hat{H}$ by

$$
\begin{aligned}
\Delta_{D}\left(i_{D}(a)\right) & =i_{D}\left(a_{(1)}\right) \otimes i_{D}\left(a_{(2)}\right) \\
\Delta_{D}(D(\varphi)) & =D\left(\varphi_{(2)}\right) \otimes D\left(\varphi_{(1)}\right) \\
S_{D}\left(i_{D}(a)\right) & =i_{D}(S(a)) \\
S_{D}(D(\varphi)) & =D\left(\hat{S}^{-1}(\varphi)\right) \\
R_{D} & =\left(i_{D} \otimes i d_{\mathcal{D}(H)}\right)(\mathbf{D})
\end{aligned}
$$

This structure may now immediately be transported to $\mathcal{M}_{R}(H)$ via $\lambda_{R}$ to give $\Delta_{M}, S_{M}$ and $R_{M}$, the formulae for which however turn out to look less transparent.

$$
\begin{aligned}
\Delta_{M} \circ i_{M} & =i_{M} \circ \Delta \\
\left(i d \otimes \Delta_{M}\right)(\mathbf{M}) & =\mathbf{R}_{o p}^{12} \mathbf{M}^{13}\left(\mathbf{R}_{o p}^{12}\right)^{-1} \mathbf{M}^{12} \\
S_{M} \circ i_{M} & =i_{M} \circ S \\
\left(i d \otimes S_{M}\right)(\mathbf{M}) & =\left(S^{-1} \otimes i d\right)\left(\mathbf{R}_{o p}^{-1} \mathbf{M R}_{o p}\right) \\
R_{M} & =\left(i_{M} \otimes i d_{\mathcal{M}_{R}(H)}\right)\left(\mathbf{R}_{o p}^{-1} \mathbf{M}\right)
\end{aligned}
$$

where $\mathbf{R}_{o p}$ and $\mathbf{M}$ have the same meaning as in (5.7). As it is explained at the end of this section these results correct some inconsistencies in [AFFS].

We now skip the intermediate object $\mathcal{M}_{R}(H)$ and study immediately the composition $\Lambda_{R}=$ $\pi_{R} \circ \lambda_{R}$. To show that up to cocycle equivalence $\Lambda_{R}$ provides a Hopf algebra map we first recall the natural coproduct $\Delta_{H \otimes H}$ given on $H \otimes_{\text {alg }} H$ by

$$
\Delta_{H \otimes H}(a \otimes b):=\left(a_{(1)} \otimes b_{(1)}\right) \otimes\left(a_{(2)} \otimes b_{(2)}\right)
$$

Lemma 7.1: Let $T:=R_{23}^{-1} \in(H \otimes H) \otimes(H \otimes H)$ ๆ. Then $T$ is a left $\Delta_{H \otimes H^{-c o c y c l e} \text { and }}$ therefore

$$
\delta_{H \otimes H}:=A d T \circ \Delta_{H \otimes H}
$$

is also coassociative.

Proof: Putting $R^{-1}=\sum_{i} u^{i} \otimes v^{i}$ we have in $(H \otimes H)^{\otimes 3}$

$$
\begin{aligned}
T^{12}\left(\Delta_{H \otimes H} \otimes i d\right)(T) & =R_{23}^{-1} \sum\left[\left(\mathbf{1} \otimes u_{(1)}^{i}\right) \otimes\left(\mathbf{1} \otimes u_{(2)}^{i}\right) \otimes\left(v^{i} \otimes \mathbf{1}\right)\right] \\
& =R_{23}^{-1} R_{45}^{-1} R_{25}^{-1} \\
& =R_{45}^{-1} R_{23}^{-1} R_{25}^{-1} \\
& =R_{45}^{-1} \sum\left[\left(\mathbf{1} \otimes u^{i}\right) \otimes\left(v_{(1)}^{i} \otimes \mathbf{1}\right) \otimes\left(v_{(2)}^{i} \otimes \mathbf{1}\right)\right] \\
& =T^{23}\left(i d \otimes \Delta_{H \otimes H}\right)(T)
\end{aligned}
$$

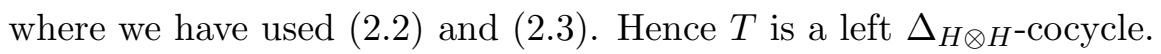

\footnotetext{
${ }^{5}$ From now on lower indices refer to the embedding $H \otimes H \rightarrow H^{\otimes n}, n \geq 3$, and upper indices - as before refer to the embeddings $\mathcal{A} \otimes \mathcal{A} \rightarrow \mathcal{A}^{\otimes 3}$ or $H \otimes \mathcal{A} \rightarrow \mathcal{H} \otimes \mathcal{A} \otimes \mathcal{A}$, respectively, where $\mathcal{A}=H \otimes H$. Also, from now on $\otimes$ always means $\otimes_{\text {alg }}$.
} 
Replacing $(\Delta, R)$ by $\left(\Delta_{o p}, R_{o p}\right)$, the coproduct $\delta_{H \otimes H}$ has already appeared in Theorem 2.7 of [RS1] see also 7.3 of [M8]. Next, we have

Lemma 7.2 Let $\mathcal{R}_{H \otimes H} \in(H \otimes H) \otimes(H \otimes H)$ be given by

$$
\mathcal{R}_{H \otimes H}:=R_{41}^{-1} R_{42}^{-1} R_{13} R_{23}
$$

Then $\mathcal{R}_{H \otimes H}$ is quasi-triangular with respect to $\delta_{H \otimes H}$.

Proof: Since $R$ and $R_{o p}^{-1}$ are quasi-triangular w.r.t. $\Delta, \mathcal{R}_{H \otimes H}^{\prime}:=R_{42}^{-1} R_{13} \in(H \otimes H) \otimes(H \otimes H)$ is quasitriangular w.r.t. $\Delta_{H \otimes H}$. The claim now follows from the twist equivalence

$$
\mathcal{R}_{H \otimes H}=T_{o p} \mathcal{R}_{H \otimes H}^{\prime} T^{-1}
$$

We are now in the position to prove that $\Lambda_{R}$ provides a homomorphism of quasitriangular Hopf algebras $\left(\mathcal{D}(H), R_{D}, \Delta_{D}\right) \rightarrow\left(H \otimes H, \delta_{H \otimes H}, \mathcal{R}_{H \otimes H}\right)$, which in fact coincides with the homomorphism given in Theorem 2.9 of [RS1], see also Chapter 7 of [M8].

Theorem 7.3 For any quasitriangular $R \in H \otimes H$ denote $\Lambda_{R}:=\pi_{R} \circ \lambda_{R}: \mathcal{D}(H) \rightarrow H \otimes H$. Then

$$
\begin{aligned}
& \text { i) } \quad \Lambda_{R} \circ i_{D}=\Delta \\
& \text { ii) } \quad\left(i d \otimes \Lambda_{R}\right)(\mathbf{D})=R_{31}^{-1} R_{12} \\
& \text { iii) } \quad \delta_{H \otimes H} \circ \Lambda_{R}=\left(\Lambda_{R} \otimes \Lambda_{R}\right) \circ \Delta_{D} \\
& \text { iv) } \quad\left(\Lambda_{R} \otimes \Lambda_{R}\right)\left(R_{D}\right)=\mathcal{R}_{H \otimes H}
\end{aligned}
$$

Moreover, $\Lambda_{R}$ is uniquely determined by i) and ii) and $\Lambda_{R}$ is an isomorphism if and only if $\operatorname{mon}_{R}$ is bijective.

Proof: Part i) follows from Theorem 6.4i). To prove ii) we use Theorem 6.4ii), (5.7) and (2.2) to compute

$$
\left(i d \otimes \Lambda_{R}\right)(\mathbf{D})=(i d \otimes \Delta)\left(R_{o p}^{-1}\right) R_{21} R_{12}=R_{31}^{-1} R_{12}
$$

The uniqueness of $\Lambda_{R}$ under conditions i) and ii) follows from Lemma 5.2. Next, using i), the claim iii) holds on $i_{D}(H) \subset \mathcal{D}(H)$ since

$$
\delta_{H \otimes H}(\Delta(a))=R_{23}^{-1}\left(a_{(1)} \otimes a_{(3)} \otimes a_{(2)} \otimes a_{(4)}\right) R_{23}=\Delta\left(a_{(1)}\right) \otimes \Delta\left(a_{(2)}\right)
$$

It remains to check that iii) holds on all $D(\varphi), \varphi \in \hat{H}$, where $\Delta_{D}$ is given by $\hat{\Delta}_{o p}$, according to (7.2). Hence, by (2.12) we have to show

$$
\mathbf{F}_{H \otimes H}^{13} \mathbf{F}_{H \otimes H}^{12}=\left(i d \otimes \delta_{H \otimes H}\right)\left(\mathbf{F}_{H \otimes H}\right) \in H \otimes(H \otimes H)^{\otimes 2}
$$

where $\mathbf{F}_{H \otimes H}=\left(i d \otimes \Lambda_{R}\right)(\mathbf{D}) \in H \otimes(H \otimes H)$. Using (7.14) we have

$$
\mathbf{F}_{H \otimes H}^{13} \mathbf{F}_{H \otimes H}^{12}=R_{51}^{-1} R_{14} R_{31}^{-1} R_{12}
$$

and

$$
\begin{aligned}
\left(i d \otimes \delta_{H \otimes H}\right)\left(\mathbf{F}_{H \otimes H}\right) & =R_{34}^{-1} \sum\left(v^{i} x^{j} \otimes y_{(1)}^{j} \otimes u_{(1)}^{i} \otimes y_{(2)}^{j} \otimes u_{(2)}^{i}\right) R_{34} \\
& =R_{34}^{-1} R_{51}^{-1} R_{31}^{-1} R_{14} R_{12} R_{34} \\
& =R_{51}^{-1}\left(R_{34}^{-1} R_{31}^{-1} R_{14} R_{34}\right) R_{12} \\
& =R_{51}^{-1} R_{14} R_{31}^{-1} R_{12} \\
& =\mathbf{F}_{H \otimes H}^{13} \mathbf{F}_{H \otimes H}^{12}
\end{aligned}
$$


where in the second line we have used (2.2), (2.3) and in the fourth line the Yang-Baxter equations in the form

$$
R_{14} R_{34} R_{31}=R_{31} R_{34} R_{14}
$$

which follow from $(2.2)-(2.5)$. This proves $(7.15)$ and therefore iii). To prove iv) we use (7.5), i) and ii) to get

$$
\begin{aligned}
\left(\Lambda_{R} \otimes \Lambda_{R}\right)\left(R_{D}\right) & =\Delta\left(e_{\nu}\right) \otimes\left(e^{\nu} \otimes i d \otimes i d\right)\left(R_{31}^{-1} R_{12}\right) \\
& =(\Delta \otimes i d \otimes i d)\left(R_{31}^{-1} R_{12}\right) \\
& =R_{41}^{-1} R_{42}^{-1} R_{13} R_{23} \\
& =\mathcal{R}_{H \otimes H}
\end{aligned}
$$

Let me close by pointing out that [AFFS] seem to provide a coproduct $\Delta_{\mathcal{K}}$ on $\mathcal{K} \equiv \mathcal{M}_{R}(H)$ such that

$$
\Delta_{H \otimes H}^{\prime}:=\left(\pi_{R} \otimes \pi_{R}\right) \circ \Delta_{\mathcal{K}} \circ \pi_{R}^{-1}
$$

satisfies

$$
\Delta_{H \otimes H}^{\prime}(a \otimes \mathbf{1})=\left(a_{(1)} \otimes \mathbf{1}\right) \otimes\left(a_{(2)} \otimes \mathbf{1}\right) .
$$

Indeed, assuming $\pi_{R}$ invertible and defining as in [AFFS]

$$
\begin{aligned}
& \mathbf{M}_{+}:=\left(i d_{H} \otimes \pi_{R}^{-1}\right)\left(R_{o p} \otimes \mathbf{1}_{H}\right) \in H \otimes \mathcal{M}_{R}(H) \\
& \mathbf{M}_{-}:=\left(i d_{H} \otimes \pi_{R}^{-1}\right)\left(R^{-1} \otimes \mathbf{1}_{H}\right) \in H \otimes \mathcal{M}_{R}(H)
\end{aligned}
$$

equ. $(\overline{7.16})$ would be equivalent to

$$
\left(i d_{H} \otimes \Delta_{\mathcal{K}}\right)\left(\mathbf{M}_{ \pm}\right)=\mathbf{M}_{ \pm}^{12} \mathbf{M}_{ \pm}^{13}
$$

or

$$
\left(i d_{H} \otimes \Delta_{\mathcal{K}}\right)(\mathbf{M})=\mathbf{M}_{+}^{12} \mathbf{M}^{13}\left(\mathbf{M}_{-}^{12}\right)^{-1}
$$

which are the formulas given in Section 4 of [AFFS]. On the other hand, equ. (4.15) of [AFFS] implies

$$
\Delta_{H \otimes H}^{\prime}\left(a_{(1)} \otimes a_{(2)}\right)=\left(a_{(1)} \otimes a_{(2)}\right) \otimes\left(a_{(3)} \otimes a_{(4)}\right)
$$

which would be consistent with our $\delta_{H \otimes H}$. However, equ. (7.16) is manifestly inconsistent with (7.21). In fact, writing

$$
(\mathbf{1} \otimes a)=\left(S\left(a_{(1)}\right) \otimes \mathbf{1}\right)\left(a_{(2)} \otimes a_{(3)}\right)
$$

it is easy to see that a map $\Delta_{H \otimes H}^{\prime}: H \otimes H \rightarrow(H \otimes H) \otimes(H \otimes H)$ obeying (7.16) and (7.21) cannot consistently be extended to an algebra homomorphism.

After presenting these results I have been informed [S] that there will be a revised version of [AFFS] reproducing $\delta_{H \otimes H}$ - or equivalently $\Delta_{M}$ given in $(7.6),(7.7)$ - at least up to cocycle equivalence. 


\section{A Multi-Loop Algebras}

Having identified the monodromy algebra $\hat{H}_{R}$ as a braided group we show in this Appendix that the multi-loop algebras of [AS] arise as braided tensor products of braided groups in the sense of Majid [M4,M7]. We recall that these are defined in the natural way so as to obtain all structural maps as $H$-module morphisms with respect to the coadjoint action $\triangleright$. More generally one has

Definition A.1 [M4, M7] Let $\left(H, \Delta, R=\sum x^{i} \otimes y^{i}\right)$ be a quasitriangular Hopf algebra and let $\mathcal{A}$ and $\mathcal{B}$ be left $H$-module algebras with both actions denoted by $\triangleright$. The braided tensor product algebra $\mathcal{A} \otimes_{R} \mathcal{B}$ is defined to be the vector space $\mathcal{A} \otimes \mathcal{B}$ with multiplication given for $a, a^{\prime} \in \mathcal{A}$ and $b, b^{\prime} \in \mathcal{B}$ by

$$
\left(a \otimes_{R} b\right)\left(a^{\prime} \otimes_{R} b^{\prime}\right):=\sum a\left(y^{i} \triangleright a^{\prime}\right) \otimes_{R}\left(x^{i} \triangleright b\right) b^{\prime}
$$

One immediately checks that $\mathcal{A} \otimes_{R} \mathcal{B}$ is a again an $H$-module algebra with action

$$
h \triangleright\left(a \otimes_{R} b\right):=\left(h_{(1)} \triangleright a\right) \otimes_{R}\left(h_{(2)} \triangleright b\right)
$$

Moreover, the braided tensor product is associative in the sense that $\left(\mathcal{A}_{1} \otimes_{R} \mathcal{A}_{2}\right) \otimes_{R} \mathcal{A}_{3}$ and $\mathcal{A}_{1} \otimes_{R}\left(\mathcal{A}_{2} \otimes_{R} \mathcal{A}_{3}\right)$ define the same algebra structure on $\mathcal{A}_{1} \otimes \mathcal{A}_{2} \otimes \mathcal{A}_{3}$.

We now apply this to the multi-loop algebras introduced in Section 6 of [AS] and show that with respect to the coadjoint action they are indeed the $m$-fold braided tensor product of the associated one-loop (三 monodromy) algebras. To this end let us denote

$$
\mathcal{L}_{m}:=\hat{H}_{R} \otimes_{R} \cdots \otimes_{R} \hat{H}_{R}
$$

the $m$-fold braided tensor product and let $\mathbf{M}_{\nu}, 1 \leq \nu \leq m$, denote the $m$ copies of monodromy matrices in $H \otimes \mathcal{L}_{m}$

$$
\mathbf{M}_{\nu}=\left(i d_{H} \otimes \iota_{\nu}\right)(\mathbf{M})
$$

where $\iota_{\nu}: \hat{H}_{R} \rightarrow \mathcal{L}_{m}$ is the obvious embedding into the $\nu$-th tensor factor. We then have

Proposition A.2 For $\mu<\nu$ the following relations hold in $H \otimes H \otimes \mathcal{L}_{m}$

$$
\mathbf{M}_{\nu}^{13} R^{12} \mathbf{M}_{\mu}^{23}=R^{12} \mathbf{M}_{\mu}^{23}\left(R^{-1}\right)^{12} \mathbf{M}_{\nu}^{13} R^{12}
$$

Proof: We only proof the case $m=2$, from which the general case follows straight forwardly. Putting $M_{\nu}(\varphi):=(\varphi \otimes i d)\left(\mathbf{M}_{\nu}\right), \varphi \in \hat{H}$, we conclude from (A.1)

$$
M_{2}(\psi) M_{1}(\xi)=\sum_{i} M_{1}\left(y^{i} \triangleright \xi\right) M_{2}\left(x^{i} \triangleright \psi\right)
$$

which by 3.2 implies

$$
\begin{aligned}
\mathbf{M}_{2}^{13} \mathbf{M}_{1}^{23} & =\sum_{i}\left[S\left(x_{(1)}^{i}\right) \otimes S\left(y_{(1)}^{i}\right) \otimes \mathbf{1}_{\mathcal{A}}\right] \mathbf{M}_{1}^{23} \mathbf{M}_{2}^{13}\left[x_{(2)}^{i} \otimes y_{(2)}^{i} \otimes \mathbf{1}_{\mathcal{A}}\right] \\
& =\sum_{i, j, k, \ell}\left[S\left(x^{i} x^{j}\right) \otimes S\left(y^{j} y^{\ell}\right) \otimes \mathbf{1}_{\mathcal{A}}\right] \mathbf{M}_{1}^{23} \mathbf{M}_{2}^{13}\left[x^{k} x^{\ell} \otimes y^{i} y^{k} \otimes \mathbf{1}_{A}\right] \\
& =\sum_{\ell}\left[\mathbf{1}_{H} \otimes S\left(y^{\ell}\right) \otimes \mathbf{1}_{\mathcal{A}}\right] R^{12} \mathbf{M}_{1}^{23}\left(R^{-1}\right)^{12} \mathbf{M}_{2}^{13} R^{12}\left[x^{\ell} \otimes \mathbf{1}_{H} \otimes \mathbf{1}_{\mathcal{A}}\right]
\end{aligned}
$$


from which the claim follows as in the proof of Proposition 2.4. Here we have used (2.2) and $(2.3)$ in the second line and the identities $(S \otimes i d)(R)=R^{-1}$ and $(S \otimes S)(R)=R$ in the last line.

A comparison with equ.(6.1) of [AS] shows that up to an ordering convention our definition of $\mathcal{L}_{m}$ coincides with their multiloop algebras.

Next we note that the monodromy homomorphism mon $_{R}$ naturally extends to multi-loop algebras. As a matter of fact, being an $H$-module map intertwining the coadjoint action on $\hat{H}_{R}$ with the adjoint action on $H$ according to Proposition 6.1 , we can use its ordinary $m$-fold tensor product $\operatorname{mon}_{R}{ }^{\otimes m}=\operatorname{mon}_{R} \otimes \cdots \otimes \operatorname{mon}_{R}$ to obtain an $H$-module algebra morphism

$$
\operatorname{mon}_{R}^{\otimes m}: \mathcal{L}_{m} \rightarrow H \otimes_{R} \cdots \otimes_{R} H
$$

where on the r.h.s. the braided tensor product with respect to the left adjoint action of $H$ on itself is understood.

Next, similarly as crossed products with respect to inner actions are isomorphic to algebraic tensor products (see e.g. Lemma 6.3), we now have the following "bosonization formula".

Proposition A.3 Consider $H$ as a left $H$-module algebra under the adjoint action and let $\iota_{\mathcal{A}}: H \rightarrow \mathcal{A}$ be a unital algebra map inducing an inner left $H$-action $\triangleright$ on $\mathcal{A}$ by

$$
h \triangleright a:=\iota_{\mathcal{A}}\left(h_{(1)}\right) a \iota_{\mathcal{A}}\left(S\left(h_{(2)}\right)\right), h \in H, a \in \mathcal{A} .
$$

Then the linear map $V_{\mathcal{A}}: \mathcal{A} \otimes_{R} H \rightarrow \mathcal{A} \otimes_{\text {alg }} H$

$$
V_{\mathcal{A}}\left(a \otimes_{R} h\right):=\sum_{i, j} a \iota_{\mathcal{A}}\left(y^{i} y^{j}\right) \otimes_{a l g} x^{i} h S\left(x^{j}\right)
$$

defines an algebra isomorphism satisfying for all $a \in \mathcal{A}$ and $h, h^{\prime} \in H$

$$
V_{\mathcal{A}}\left(h \triangleright\left(a \otimes_{R} h^{\prime}\right)\right)=\Delta_{\mathcal{A}}\left(h_{(1)}\right) V_{\mathcal{A}}\left(a \otimes_{R} h^{\prime}\right) \Delta_{\mathcal{A}}\left(S\left(h_{(2)}\right)\right)
$$

where $\Delta_{\mathcal{A}}:=\left(\iota_{\mathcal{A}} \otimes i d_{H}\right) \circ \Delta$.

Proof: Using $(S \otimes i d)(R)=R^{-1}$ and (2.14) the inverse of $V_{\mathcal{A}}$ is given by

$$
V_{\mathcal{A}}^{-1}\left(a \otimes_{a l g} h\right)=\sum_{i, j} a \iota_{\mathcal{A}}\left(y^{j} S\left(y^{i}\right)\right) \otimes_{R} x^{i} h x^{j}
$$

Now $V_{\mathcal{A}}\left(a \otimes_{R} \mathbf{1}_{H}\right)=\left(a \otimes_{\text {alg }} \mathbf{1}_{H}\right)$ and

$$
V_{\mathcal{A}}\left(\mathbf{1}_{\mathcal{A}} \otimes_{R} h\right)=\left(\iota_{\mathcal{A}} \otimes i d\right)\left(R_{o p}\left(\mathbf{1}_{H} \otimes_{\text {alg }} h\right) R_{o p}^{-1}\right) .
$$

proving that the restrictions of $V_{\mathcal{A}}$ to the subalgebras $\mathcal{A} \otimes_{R} \mathbf{1}_{H}$ and $\mathbf{1}_{\mathcal{A}} \otimes_{R} H$ are algebra maps. We are left to check that $V_{\mathcal{A}}$ respects the commutation relations between $\mathcal{A} \otimes_{R} \mathbf{1}_{H}$ and $\mathbf{1}_{\mathcal{A}} \otimes_{R} H$. To this end we compute (implying a summation convention over doubled indices)

$$
\begin{aligned}
V_{\mathcal{A}}\left(\left(\mathbf{1}_{\mathcal{A}} \otimes_{R} h\right)\left(a \otimes_{R} \mathbf{1}_{H}\right)\right) & =V_{\mathcal{A}}\left(y^{i} \triangleright a \otimes_{R} x^{i} \triangleright h\right) \\
& =y_{(1)}^{i} a S\left(y_{(2)}^{i}\right) y^{m} y^{n} \otimes_{a l g} x^{m} x_{(1)}^{i} h S\left(x_{(2)}^{i}\right) S\left(x^{n}\right) \\
& =y^{j} y^{\ell} a S\left(y^{i} y^{k}\right) y^{m} y^{n} \otimes_{a l g} x^{m} x^{i} x^{j} h S\left(x^{n} x^{k} x^{\ell}\right) \\
& =y^{j} y^{\ell} a \otimes_{a l g} x^{j} h S\left(x^{\ell}\right) \\
& =V_{\mathcal{A}}\left(\mathbf{1}_{\mathcal{A}} \otimes_{R} h\right) V_{\mathcal{A}}\left(a \otimes_{R} \mathbf{1}_{H}\right)
\end{aligned}
$$


where we have used (2.2) and (2.3) in the third line and (2.14) in the fourth line, and where by a convient abuse of notation we have dropped the symbol $\iota_{\mathcal{A}}$. This proves that $V_{\mathcal{A}}: \mathcal{A} \otimes_{R} H \rightarrow$ $\mathcal{A} \otimes_{\text {alg }} H$ provides an algebra map. To prove (A.9) we use that the general definition (A.2) provides a Hopf module action. Hence, it is enough to check (A.9) separately on the generating factors $\mathcal{A} \otimes_{R} \mathbf{1}_{H} \cong \mathcal{A}$ and $\mathbf{1}_{\mathcal{A}} \otimes_{R} H \cong H$. Now, dropping again the symbol $\iota_{\mathcal{A}}$ we have in $\mathcal{A} \otimes_{\text {alg }} H$

$$
\begin{aligned}
\Delta_{\mathcal{A}}\left(h_{(1)}\right)\left(a \otimes_{\text {alg }} \mathbf{1}_{H}\right) \Delta_{\mathcal{A}}\left(S\left(h_{(2)}\right)\right) & =h_{(1)} a S\left(h_{(4)}\right) \otimes_{\text {alg }} h_{(2)} S\left(h_{(3)}\right) \\
& =(h \triangleright a) \otimes_{\text {alg }} \mathbf{1}_{H}
\end{aligned}
$$

proving $(\underline{\mathrm{A.9}})$ on $\left(\mathcal{A} \otimes_{R} \mathbf{1}_{H}\right)$. On $\left(\mathbf{1}_{\mathcal{A}} \otimes_{R} H\right)$ we get

$$
\begin{aligned}
V_{\mathcal{A}}\left(\mathbf{1}_{\mathcal{A}} \otimes_{R} h \triangleright h^{\prime}\right) & =y^{i} y^{j} \otimes_{\text {alg }} x^{i} h_{(1)} h^{\prime} S\left(h_{(2)}\right) S\left(x^{j}\right) \\
& =y^{i} y^{j} h_{(3)} S\left(h_{(4)}\right) \otimes_{\text {alg }} x^{i} h_{(1)} h^{\prime} S\left(x^{j} h_{(2)}\right) \\
& =h_{(1)} y^{i} y^{j} S\left(h_{(4)}\right) \otimes_{\text {alg }} h_{(2)} x^{i} h^{\prime} S\left(x^{j}\right) S\left(h_{(3)}\right) \\
& =\Delta_{\mathcal{A}}\left(h_{(1)}\right) V_{\mathcal{A}}\left(\mathbf{1}_{\mathcal{A}} \otimes_{R} h^{\prime}\right) \Delta_{\mathcal{A}}\left(S\left(h_{(2)}\right)\right)
\end{aligned}
$$

This proves (A.9) and therefore Proposition A.3.

Corollary A.4 Under the conditions of Proposition A.3 put $\delta_{\mathcal{A}}:=V_{\mathcal{A}}^{-1} \circ \Delta_{\mathcal{A}}: H \rightarrow \mathcal{A} \otimes_{R} H$. Then $\delta_{\mathcal{A}}$ implements the $H$-action on $\mathcal{A} \otimes_{R} H$, i.e.

$$
h \triangleright\left(a \otimes_{R} h^{\prime}\right)=\delta_{\mathcal{A}}\left(h_{(1)}\right)\left(a \otimes_{R} h^{\prime}\right) \delta_{\mathcal{A}}\left(S\left(h_{(2)}\right)\right)
$$

Note that we may in particular put $\mathcal{A}=H$ and $\iota_{\mathcal{A}}=i d$ to obtain, using $(S \otimes S)(R)=R$

$$
\begin{aligned}
\delta_{\mathcal{A}}(a) & =\sum_{i, j} y^{j} a_{(2)} y^{i} \otimes S^{-1}\left(x^{i}\right) x^{j} a_{(1)} \\
& =\sum_{i, j} a_{(1)} y^{j} S\left(y^{i}\right) \otimes x^{i} a_{(2)} x^{j}
\end{aligned}
$$

which coincides with $\Delta_{R}$ given in (2.1) up to a change of conventions (i.e. replacing $(H, \Delta, S, R)$ by $\left(H, \Delta_{o p}, S^{-1}, R_{o p}\right)$. This shows that $\Delta_{R}$ provides an algebra map $H \rightarrow H_{c o p} \otimes_{R_{o p}} H_{c o p}$ as remarked after Lemma 2.2.

We now put $\iota_{\mathcal{A} \otimes_{R} H}=\delta_{\mathcal{A}}$ and proceed inductively to get isomorphisms

$$
V_{\mathcal{A}, m}: \mathcal{A} \otimes_{R} H \otimes_{R} \cdots \otimes_{R} H \rightarrow \mathcal{A} \otimes_{\text {alg }} H \otimes_{\text {alg }} \cdots \otimes_{\text {alg }} H
$$

where the tensor factors $H$ appear $m$-times. Choosing in particular $\mathcal{A}=\mathbf{C}$ and $\iota_{\mathcal{A}}=\varepsilon$ and denoting $V_{\mathbf{C}, m} \equiv V_{m}$ we have proven

Theorem A.5 The map

$$
\operatorname{mon}_{R, m}:=V_{m} \circ \operatorname{mon}_{R}^{\otimes m}: \mathcal{L}_{m} \rightarrow H^{\otimes m}
$$

provides a homomorphism of algebras such that

$$
\operatorname{mon}_{R, m}(h \triangleright \underline{a})=\Delta^{(m)}\left(h_{(1)}\right) \operatorname{mon}_{R, m}(\underline{a}) \Delta^{(m)}\left(S\left(h_{(2)}\right)\right)
$$


where $\underline{a} \in \mathcal{L}_{m}, h \in H$ and $\Delta^{(m)}: H \rightarrow H^{\otimes m}$ denotes the $m$-fold coproduct $[$. Moreover, $\operatorname{mon}_{R, m}$ is an isomorphism if and only if $\operatorname{mon}_{R}$ is bijectiv.

Generalizing Lemma 6.3 in the obvious way we further have $H^{\otimes m_{\rtimes}} A d \cong H^{\otimes(m+1)}$ and therefore also a homomorphism

$$
\operatorname{Mon}_{R, m}: \mathcal{L}_{m} \rtimes H \rightarrow H^{\otimes(m+1)},
$$

which is bijective iff $\operatorname{mon}_{R}$ is bijective.

Theorem A.5 explains the representation theory of $\mathcal{L}_{m}$ given by [AS] without having to rely on any semi-simplicity assumptions. To see this explicitly we show

Proposition A.6 Let $\mathbf{M}_{\nu}=\left(i d_{H} \otimes \iota_{\nu}\right)(\mathbf{M}) \in H \otimes \mathcal{L}_{m}, 1 \leq \nu \leq m$, be the $\nu$-th monodromy matrix. Then

$$
\left(i d_{H} \otimes \operatorname{mon}_{R, m}\right)\left(\mathbf{M}_{\nu}\right)=\mathbf{N}_{\nu} \otimes \mathbf{1}_{H}^{\otimes(m-\nu)} \in H \otimes H^{\otimes m}
$$

where

$$
\mathbf{N}_{\nu}:=\left(i d_{H} \otimes \Delta^{(\nu-1)} \otimes i d_{H}\right)\left(R^{32} R^{31} R^{13}\left(R^{-1}\right)^{32}\right) \in H \otimes H^{\otimes \nu}
$$

Proof: We proceed by induction over $m \geq 1$. The case $m=1$ holds by definition of $\operatorname{mon}_{R}$. Now suppose the claim holds for $m_{0}$ and all $1 \leq \nu \leq m_{0}$. Putting $\mathcal{A}=H \otimes_{R} \cdots \otimes_{R} H\left(m_{0}\right.$ factors) we have

$$
\operatorname{mon}_{R, m_{0}+1}=\left(V_{m_{0}} \otimes i d_{H}\right) \circ V_{\mathcal{A}} \circ \operatorname{mon}_{R}^{\otimes\left(m_{0}+1\right)}
$$

Since the restriction of $V_{\mathcal{A}}$ for $\mathcal{A} \otimes_{R} \mathbf{1}_{H}$ is the identity we get with respect to the identification $\mathcal{L}_{m} \cong \mathcal{L}_{m} \otimes_{R} \hat{\mathbf{1}} \subset \mathcal{L}_{m+1}$

$$
\operatorname{mon}_{R, m_{0}+1} \mid \mathcal{L}_{m_{0}}=\operatorname{mon}_{R, m_{0}}
$$

This proves (A.12) for $m=m_{0}+1$ and $1 \leq \nu \leq m_{0}$. For $\nu=m_{0}+1$ we use

$$
\left(i d_{H} \otimes \operatorname{mon}_{R}^{\otimes\left(m_{0}+1\right)}\right)\left(\mathbf{M}_{\nu}\right)=R^{31} R^{13} \in H \otimes_{\text {alg }}\left(\mathcal{A} \otimes_{\text {alg }} H\right)
$$

Applying $\left(i d_{H} \otimes V_{\mathcal{A}}\right)$ to A.16) we get in $H \otimes_{\text {alg }}\left(\mathcal{A} \otimes_{\text {alg }} H\right)$

$$
\left(i d_{H} \otimes V_{\mathcal{A}}\right)\left(R^{31} R^{13}\right)=\left(i d_{H} \otimes \iota_{\mathcal{A}} \otimes i d_{H}\right)\left(R^{32} R^{31} R^{13}\left(R^{32}\right)^{-1}\right)
$$

Finally, according to Corollary A.4 and the inductive definition of $V_{m}$ we have $V_{m} \circ \iota_{\mathcal{A}}=\Delta^{(m)}$, which together with (A.14), (A.16) and (A.17) proves (A.12) for $m=m_{0}$ and $\nu=m_{0}+1$.

Comparing A.12) with the representations of $\mathcal{L}_{m}$ given in Section 6 of [AS] we realize that they are in fact all of the form $\tau \circ \operatorname{mon}_{R, m}$, where $\tau \in \operatorname{Rep}\left(H^{\otimes m}\right)$.

\section{References}

[AFFS] A.Yu. Alekseev, L.D. Faddev, J. Fröhlich, V. Schomerus, Representation Theory of Lattice Current Algebras, Q-alg/9604017.

[AGS] A.Yu. Alekseev, H. Grosse, V. Schomerus, Combinatorical Quantization of the Hamiltonian Chern Simons Theory, I, II, Comm. Math. Phys. 172 (1995), 317-358, and 174 (1996), 561604.

[AS] A.Yu. Alekseev, V. Schomerus, Representation Theory of Chern Simons Observables, q$\operatorname{alg} / 9503016$

\footnotetext{
${ }^{6}$ i.e. $\Delta^{(0)}=\varepsilon, \Delta^{(1)}=i d_{H}$ and $\Delta^{(m+1)}=\left(i d^{\otimes(m-1)} \otimes \Delta\right) \circ \Delta^{(m)}$
} 
[DPR] R. Dijkgraf, V. Pasquier, P. Roche, Quasi Hopf algebras, group cohomology and orbifold models, Nucl. Phys. 18B (Proc. Suppl) (1990)60.

[Dr1] V.G. Drinfeld, Quantum groups, In: Proc. Int. Cong. Math., Berkeley, 1986, p.798.

[Dr2] V.G. Drinfeld, On Almost Cocommutative Hopf Algebras, Leningrad Math. J. 1(1990), 321.

[K] C. Kassel, Quantum Groups, Springer, New York, 1995.

[NSz] F. Nill, K. Szlachányi, Quantum Chains of Hopf Algebras with Quantum Double Cosymmetry, hep-th/95 09 100, to appear in Comm. Math. Phys.

[M1] S. Majid, Physics for algebraists: Non-cocommutative and non-commutative Hopf algebras by a bicrossed product construction, J. Algbra 130 (1990), 17-64.

[M2] S. Majid, Doubles of quasitriangular Hopf algebras, Comm. Algebra 19 (1991), 3061-3073.

[M3] S. Majid, Braided Groups and Algebraic Quantum Field Theories, Lett. Math. Phys. 22 (1991), 167-175.

[M4] S. Majid, Braided Groups, J. Pure Appl. Alg. 86 (1993), 187-221.

[M5] S. Majid, Braided Matrix Structure of the Sklynanin Algebra and of the Quantum Lorentz Group, Comm. Math. Phys. 156 (1993), 607-638.

[M6] S. Majid, q-Euclidean Space and q-Wick rotation, J. Math. Phys. 35 (1994), 5025-5034.

[M7] S. Majid, Algebras and Hopf algebras in braided categories In: Adv. in Hopf Alg.. Marcel Dekkar, Lect. Notes Pure Appl. Math. 158 (1994), 55-105, also available under q-alg 9509023.

[M8] S. Majid, Foundations of Quantum Group Theory, Cambridge University Press, 1995.

[Mü] M. Müger, Quantum Double Actions on Operator Algebras and Orbifold Quantum Field Theories, Preprint DESY 96-117.

[Ra] D.E. Radford The Structure of Hopf Algebras with a Projection, J. Alg. 85 (1985), 322-347.

[RS1] N.Yu. Reshetikhin, M.A. Semenov-Tian-Shanski, Quantum R-Matrices and Factorization Problems, J. Geom. Phys. 5 (1988), 533-550.

[RS2] N.Yu. Reshetikhin, M.A. Semenov-Tian-Shanski, Central Extensions of Quantum Current Groups, Lett. Math. Phys. 19 (1990), 133-142.

[S] V. Schomerus, private communication.

[Sw] M.E. Sweedler, Hopf algebras, Benjamin 1969.

[SzV] K. Szlachányi, P. Vecsernyés, Quantum symmetry and braid group statistics in G-spin models, Commun.Math.Phys.156, 127 (1993). 